NASA Technical Memorandum 102030

ICOMP-89-10

\title{
Nonlinear Evolution of Interacting Oblique Waves on Two-Dimensional Shear Layers
}

\author{
M.E. Goldstein \\ Lewis Research Center \\ Cleveland, Ohio
}

and

S.-W. Choi

Institute for Computational Mechanics in Propulsion

Lewis Research Center

Cleveland, Ohio

June 1989

(NASA-TH-102030) NONLINEAR EVOLUTION OF INTERACTING OBLIQUE WAVES ON TWO-DIMENSIONAL $S H E A B$ LAYERS $33 \mathrm{p}$ (ter)

C SCL 2OD
N89-24575

Unclas G3/34 0217235 
NONLINEAR EVOLUTION OF INTERACTING OBLIQUE WAVES ON

TWO-DIMENSIONAL SHEAR LAYERS

\author{
M.E. Goldstein \\ National Aeronautics and Space Administration \\ Lewis Research Center \\ Cleveland, Ohio 44135
}

and

S. -W. Choi*

Institute for Computational Mechanics in Propulsion

Lewis Research Center

Cleveland, Ohio 44135

\begin{abstract}
SUMMARY
We consider the effects of critical layer nonlinearity on spatially growing oblique instability waves on nominally two-dimensional shear layers between parallel streams. The analysis shows that three-dimensional effects cause nonlinearity to occur at much smaller amplitudes than it does in two-dimensional flows. The nonlinear instability wave amplitude is determined by an integrodifferential equation with cubic type nonlinearity. The numerical solutions to this equation are worked out and discussed in some detail. We show that they always end in a singularity at a finite downstream distance.
\end{abstract}

\title{
1. INTRODUCTION
}

External excitation of (convectively unstable) free shear layers between parallel streams produces spatially growing instability waves that are initially governed by linear dynamics for sufficiently small excitation amplitudes. While the instability amplitude continues to increase with increasing downstream distance, its local growth rate must ultimately decrease due to viscous spreading of the mean shear layer. Nonlinear effects can then become important in a "critical layer" at the transverse position where the mean flow and instability wave phase velocities are equal (once the instability wave amplitude becomes sufficiently large and its growth rate becomes sufficiently small). The unsteady critical layer flow is then governed by a nonlinear vorticity equation, while the motion outside the critical layer remains essentially linear. The external instability wave growth rate is, however, completely controlled by the nonlinear dynamics of the critical layer.

There are now too many nonlinear critical layer analyses in the literature for us to summarize here. We refer the reader to the excellent review by Maslowe (1986) for nonlinear critical layers in general and to the one by Stewartson (1981) for Rossby-wave critical layers in particular. Here it is only appropriate to mention the analyses specifically concerned with spatially evolving flows. Huerre $(1980,1987)$ considers the two-dimensional incompressible shear layer in the viscous critical layer regime where the nonlinear

*Work funded under Space Act Agreement C99066G. 
effects enter only at higher order. Huerre and Scott (1980) and Robinson (1974) consider the strongly nonlinear regime, but their choice of scaling precludes the possibility of matching their results onto the linear upstream solution.

Goldstein and Leib (1988) and Goldstein and Hultgren (1988) overcame this difficulty, but again consider only the incompressible case. They consider only a two-dimensional flow, since the two-dimensional instability wave is the most rapidly growing linear mode in that case. However, the oblique modes exhibit the most rapid growth in sufficiently high Mach-number supersonic shear layers (Gropengeisser, 1969; Jackson and Grosch, 1988). Goldstein and Leib (1989) consider the case where the unsteady flow evolves from a single oblique mode, in which case it is appropriate to suppose that the initial instability wave grows in its propagation direction. The cross flow velocity effects can then be eliminated from the analysis by use of an appropriate "Squire transform," but the critical layer nonlinearity still behaves quite differently from the incompressible case - primarily because the temperature fluctuations have algebraic singularities in the critical layer and therefore become very large relative to the remaining velocity components causing the critical layer nonlinearity to occur at a much smaller amplitude vis-a-vis the two-dimensional isothermal case. The resulting critical layer nonlinearity is then weak in the sense that the flow is governed by linear dynamics to lowest order of approximation, with nonlinearity entering only through the higher order (inhomogeneous) terms. The instability wave growth rate is still controlled by the nonlinear terms, but can now be calculated from an amplitude equation similar to the one found by Hickernell (1984) for the Rossby wave singular modes.

Here, we consider the case where the initial instability wave grows in the streamwise direction. It is then appropriate to suppose that there are two oblique modes with the same frequency and streamwise wave number but with equal and opposite (real) spanwise wave numbers in order to represent a fixed spanwise structure. (In real flows the allowable spanwise wave numbers might be selected by the side-wall positions.) The cross flow velocity fluctuations, which have the same algebraic singularity in the critical layer as the temperature fluctuations, now become coupled to the velocity fluctuations in the plane of the wave, causing the critical layer nonlinearity to again become important at smaller amplitudes than in the two-dimensional incompressible case. In fact the nonlinear oblique mode interaction causes the critical layer nonlinearity to occur at even smaller amplitudes than in the single mode compressible case (Goldstein and Leib, 1989). Critical layer nonlinearity now becomes important when the instability wave growth rate is $O\left(\varepsilon^{1 / 3}\right)$ rather than $O\left(\varepsilon^{2 / 5}\right)$, as in the Goldstein and Leib (1989) case, where $\varepsilon$ denotes the characteristic instability wave amplitude at the start of the nonlinear region.

While the phenomenon is of most importance in supersonic shear layers, we restrict the analysis to the incompressible case because the nonlinear critical layer dynamics are unaffected by compressibility effects, and their inclusion would greatly complicate the analysis. Moreover, the final amplitude equation (3.69), which is the principal result of this paper, remains valid for the compressible case. The instability amplitude outside the critical layer can again be determined from an amplitude equation because the critical layer nonlinearity is still weak. The equation is similar to that of Goldstein and Leib (1989) in that it is an integro-differential equation with cubic nonlinearity, but the structure of the nonlinear kernel function is now somewhat different from theirs. The equation still has to be solved numerically. This 
is accomplished by using a fourth-order predictor-corrector scheme to integrate in the downstream direction, starting from the upstream linear state which is prescribed far upstream in the flow (relative to the streamwise length scale of the nonlinear region).

The calculated instability wave amplitudes initially follow the prescribed linear growth, but soon begin to either saturate or increase their rate of growth when the nonlinear effects come into play. Cumulative history effects eventually reverse these trends, causing a rapid increase in amplitude which ends in a singularity at a finite downstream distance. The local asymptotic solution of the amplitude equation is derived. It. shows that the flow will become fully nonlinear everywhere in the shear layer and that the motion will then be governed by the full three-dimensional Euler's equations in the next stage of evolution downstream of the weakly nonlinear region.

The overall plan of the paper is as follows. The problem is formulated in section 2, where we show how the nonlinear critical layer gradually evolves from the strictly linear finite growth rate solution. The flow outside the critical layer is a linear, unsteady three-dimensional perturbation about the two-dimensional mean shear layer flow, which can be treated as locally parallel on the streamwise length scale over which the nonlinear effects take place. The latter occur entirely within the critical layer to the order of approximation of the analysis and determine the unknown amplitude function in the external solution. The transverse velocity fluctuation is taken as the basic variable for the external flow, and the (linear) equation for this quantity is expressed in terms of the "Squire coordinates" associated with either of the two oblique modes.

We introduce a "slowly varying" amplitude function, which depends on the streamwise coordinate through an appropriately scaled variable and is ultimately determined by the nonlinear flow in the critical layer, which we analyze in section 3. Matching with the linear external instability waves leads to the nonlinear integro-differential equation that determines the amplitude of those waves. The numerical and asymptotic solutions to this equation are discussed in section 5 .

\section{FORMULATION AND SOLUTION OUTSIDE THE CRITICAL LAYER}

We are concerned with a nearly inviscid incompressible shear flow between two parallel streams with nominally uniform velocities $U^{(1)}>U^{(2)}$. The upstream flow consists of a steady two-dimensional shear layer and a pair of oblique (i.e., three-dimensional) spatially growing (i.e., time harmonic) instability waves with the same frequency and streamwise wave numbers but with equal and opposite (real) spanwise wave numbers. The streamwise, transverse, and spanwise coordinates ( $x, y$, and $z$, respectively), the time $t$ and all velocities are normalized by $\delta_{0}, \delta_{0} / \Delta$, and $\Delta$, respectively, where $\delta_{0}$ is half the mean shear layer momentum thickness and

$$
\Delta=\frac{u^{(1)}-u^{(2)}}{2}
$$

is a measure of the velocity difference across the shear layer. When nonlinear effects do not first intervene, the gradual viscous spreading of the mean shear 
layer causes the common spatial growth rate of the two linear instability waves to gradually decrease until they approach their common neutral stability point, whose Strouhal number and streamwise and (real) spanwise wave numbers we denote by $S_{O}, \alpha$, and $\pm \beta$, respectively.

As already indicated, nonlinear effects first become important at the streamwise position (upstream of the linear neutral stability point) where the local Strouhal number $S$ (frequency normalized by $\Delta / \delta_{0}$ ) is

$$
S=S_{0}+\varepsilon^{1 / 3} S_{1}
$$

Here $S_{1}<0$ is assumed to be $O(1)$ constant, and $\varepsilon$ denotes the characteristic amplitude of the instability wave in this region. The instability wave growth rate, which is also $O\left(\varepsilon^{/ / 3}\right)$, will then be determined by the nonlinear critical layer effects. Finally, we require that the origin of the spatial coordinates $x, y, z$ be located within this nonlinear region.

The transverse velocity fluctuations of the two upstream linear instability waves will then be of the form

$$
-\bar{\alpha}\left(e^{i \beta z}+e^{-i \beta z}\right) \operatorname{Re}_{1} \alpha^{\dagger} \Phi_{1}^{(1)}(y) e^{i\left(\alpha \xi-\varepsilon{ }^{1 / 3} S_{1} t\right)-1 / 2 S_{1} U_{c}^{1} \bar{k} x_{1}},
$$

where $t$ denotes the time,

$$
\bar{\alpha} \equiv \sqrt{\alpha^{2}+\beta^{2}},
$$

$\pm \beta$ denote the (real) transverse wave numbers of each of the individual instability waves and $\pm \theta$, where

$$
\theta \equiv \tan ^{-1} \frac{\beta}{\alpha}
$$

denotes the direction of propagation (relative to the mean flow direction) of these waves at their common neutral state.

$$
\xi \equiv x-U_{c} t
$$

is a streamwise coordinate in a reference frame moving downstream with the neutral phase velocity

$$
\begin{gathered}
U_{C}=\frac{S_{0}}{\alpha}, \\
x_{1} \equiv \varepsilon^{1 / 3 x}
\end{gathered}
$$

is a scaled streamwise coordinate (in the fixed reference frame) and,

$$
\text { is }, u_{c}, \frac{\bar{\kappa}}{2}
$$

is a scaled complex wave number whose imaginary part is minus the common growth rate of the linear near neutral instability waves. $\Phi_{1}^{(1)}(y)$ is an appropriate 
solution of Rayleigh's equation, which can be taken as the neutral solution to the required order of approximation. The complex constant at is a measure of the complex (scaled) amplitude of the two waves.

The normalized pressure is denoted by $p$ and the normalized velocity components in the moving $\{\xi, y, z\}$ coordinate system by $\{u, v, w\}$. We expect the solution outside the critical layer to expand like

$$
\begin{gathered}
u=U(y)+\varepsilon u_{1}+\varepsilon^{4 / 3} u_{2}+\ldots . \\
v=\varepsilon v_{1}+\varepsilon^{4 / 3} v_{2}+\ldots \\
w=\varepsilon w_{1}+\varepsilon^{4 / 3} w_{2}+\ldots \\
p=\varepsilon p_{1}+\varepsilon^{4 / 3} p_{2}+\ldots .
\end{gathered}
$$

where $U(y)+U_{C}$ is the base-flow velocity and the form of the upstream linear solution suggests that $v_{1}$ will be of the form

$$
v_{1}=-\bar{\alpha}\left(e^{i \beta z}+e^{-i \beta z}\right) \operatorname{Re} i \Phi_{1}^{(1)}(y) A^{\dagger}\left(x_{1}\right) e^{i \alpha \zeta} \text {, }
$$

where

$$
\zeta \equiv \xi-S_{1} \varepsilon^{1 / 3} t / \alpha
$$

and $A^{\dagger}$ is a function of the slow streamwise variable $x_{1}$, which will ultimately be determined by the nonlinear flow in the critical layer, but matching with the upstream linear instability waves requires that it satisfy the upstream boundary condition

$$
A^{\dagger}-a^{\dagger} e^{-S_{1} U_{C}^{\prime} \bar{k} x_{1} / 2} \text { as } \quad x_{1} \rightarrow-\infty .
$$

The $O\left(\varepsilon^{4 / 3}\right)$ terms (in (2.8) to (2.11)) are induced by nonlinear effects within the critical layer and by derivatives of the $O(\varepsilon)$ terms with respect to $x_{1}$. They are, therefore, at least in part, associated with linear growth rate effects. In fact, the entire solution (2.9) satisfies linear dynamics to the indicated order and is therefore determined by Rayleigh's equation

$$
\left(\frac{\partial}{\partial t}+U \frac{\partial}{\partial \xi}\right) \nabla^{2} v-U^{\prime \prime} \frac{\partial v}{\partial \xi}=0 \text {, }
$$

where the primes denote differentiation with respect to $y$ and

$$
\nabla^{2} \equiv \frac{\partial^{2}}{\partial \xi^{2}}+\frac{\partial^{2}}{\partial y^{2}}+\frac{\partial^{2}}{\partial z^{2}}
$$

is the Laplacian in the moving reference frame. It follows that $v_{2}$ is a function of the form $v_{2}\left(\zeta, x_{1}, y, z\right)$ and therefore satisfies 


$$
\left(U \nabla^{2}-U^{\prime \prime}\right) \frac{\partial v_{2}}{\partial \zeta}=-\left(2 U \frac{\partial^{2}}{\partial \zeta^{2}}-U^{\prime \prime}\right) \frac{\partial v_{1}}{\partial x_{1}}+\left[\frac{S_{1}}{\alpha} \frac{\partial}{\partial \zeta}-\left(U+U_{c}\right) \frac{\partial}{\partial x_{1}}\right] \nabla^{2} v_{1},
$$

where $\nabla^{2}$ now denotes the Laplacian with respect to $\zeta, y$, and $z$ and the partial derivatives with respect to $\zeta$ are at constant $x_{1}$ and vice-versa. It follows from continuity that

$$
\frac{\partial u_{2}}{\partial \zeta}+\frac{\partial v_{2}}{\partial y}+\frac{\partial w_{2}}{\partial z}+\frac{\partial u_{1}}{\partial x_{1}}=0
$$

$\Phi_{1}^{(1)}$ must, of course, satisfy the reduced Rayleigh's equation

$$
L^{(1)} \Phi_{1}^{(1)}=0
$$

where

$$
L^{(1)} \equiv \frac{d^{2}}{d y^{2}}-\left(\bar{\alpha}^{2}+\frac{U^{\prime \prime}}{U}\right)
$$

denotes the reduced Rayleigh's operator, and $u_{1}$ and $w_{1}$ are then given by

$$
\begin{array}{r}
u_{1}=\left(e^{i \beta z}+e^{-i \beta z}\right) \cos \theta \operatorname{Re}\left[\Phi_{1}^{(1)^{\prime}}(y)+\tan ^{2} \theta \frac{U^{\prime}(y)}{U(y)} \Phi_{1}^{(1)}(y)\right] A^{\dagger}\left(x_{1}\right) e^{i \alpha \zeta} \\
+\operatorname{Re}_{1 \pm}^{(0)}\left(y_{1} x_{1}\right) e^{2 i \beta z},
\end{array}
$$

and

$$
w_{1}=-2 \sin \theta \sin \beta z \operatorname{Re} i\left(\frac{U^{\prime}}{U} \Phi_{1}^{(1)}-\Phi^{(1)^{\prime}}\right) A^{\dagger} e^{i \alpha \zeta}
$$

where the $\zeta$-independent term in $(2.21)$, which is governed by linear dynamics in the main shear layer, is induced by nonlinear effects in the critical layer.

Then since $u_{2}, v_{2}$, and $w_{2}$ have the same $\zeta$-periodicity as $v_{1}$, they must be expressible in the form

$$
\begin{gathered}
u_{2}=\operatorname{Re} \sum_{m=0}^{\infty} F_{2}^{(m)}\left(y, z, x_{1}\right) e^{i m \alpha \zeta} \\
v_{2}=-\bar{\alpha} \operatorname{Re} i \sum_{m=0}^{\infty} \tilde{\Phi}_{2}^{(m)}\left(y, z, x_{1}\right) e^{i m \alpha \zeta} \\
w_{2}=\operatorname{Re} \sum_{m=0}^{\infty} H_{2}^{(m)}\left(y, z, x_{1}\right) e^{i m \alpha \zeta}
\end{gathered}
$$


Substituting (2.12) and (2.24) into (2.17) we find that

$$
\tilde{\Phi}_{2}^{(1)}=2 \cos \beta z \Phi_{2}^{(1)}\left(y, x_{1}\right) \text {. }
$$

where $\Phi_{2}^{(1)}$ satisfies

$$
i \alpha U L^{(1)} \Phi_{2}^{(1)}=\left[\left(i S_{1}-U_{c} \frac{d}{d x_{1}}\right) A^{\dagger}\right]\left(\Phi_{1}^{(1) "}-\alpha^{-2} \Phi_{1}^{(1)}\right)+2 \alpha^{2} U \frac{d A^{\dagger}}{d x_{1}} \Phi_{1}^{(1)}
$$

It now follows from $(2.23)$ to $(2.25)$ and $(2.18)$ that

$$
F_{2}^{(1)}=\left(\sec \theta \bar{F}_{2}^{(1)}+\sin \theta \tan \theta \bar{H}_{2}^{(1)}\right)\left(e^{i \beta z}+e^{-i \beta z}\right) \text {, }
$$

and

$$
H_{2}^{(1)}=-\sin \theta \bar{H}_{2}^{(1)}\left(e^{i \beta z}-e^{-i \beta z}\right) \text {, }
$$

where $\bar{F}_{2}^{(1)}$ is given by

$$
\bar{F}_{2}^{(1)}=\Phi_{2}^{(1)^{\prime}}-\frac{\cos ^{2} \theta}{i \alpha}\left(\Phi_{1}^{(1)^{\prime}}+\tan ^{2} \theta \frac{U^{\prime}}{U} \Phi_{1}^{(1)}\right) \frac{d A^{\dagger}}{d x_{1}}
$$

and $\bar{H}_{2}$ can be defined similarly, but will not be needed in the following analysis.

Equations (2.19) and (2.27) must, in general, be solved numerically. Fortunately, we need only know the local behavior of their solutions at the critical level where $U(y)=0$. This will occur at the inflection point $U^{\prime \prime}\left(y_{c}\right)=0$, or

$$
U_{C}^{\prime \prime}=0,
$$

(where the subscript $c$ is used to denote quantities at the critical level) provided we assume, as we now do, that $U(y)$ has only a single inflection point.

The critical level, which we can always suppose to lie at $y=0$, is then a regular point for the operator (2.20) and equation (2.19) will, therefore, possess the two linearly independent solutions

$$
\tilde{\Phi}^{(1)}=1+\frac{1}{2 !}\left(\bar{\alpha}^{2}+\frac{U_{c}^{\prime}{ }^{\prime}}{U_{c}^{\prime}}\right) y^{2}+\ldots .
$$


and

$$
\tilde{\Phi}^{(2)}=y+\frac{1}{3 !}\left(\bar{\alpha}^{2}+\frac{U_{c}^{\prime \prime}}{U_{c}^{\prime}}\right) y^{3}+\ldots \cdot
$$

as $y \rightarrow 0$.

Then we can put

$$
\Phi_{1}^{(1)}=\tilde{\Phi}^{(1)}+b_{1} \tilde{\Phi}^{(2)} \text {, }
$$

where $b_{1}$ is a constant which must, in general, be determined along with $\bar{\alpha}$ by solving (2.19) numerically subject to the proper boundary conditions at $y= \pm \infty$. Then $(2.21)$ and (2.22) imply that

$$
\begin{aligned}
& u_{1}=\cos \theta\left(e^{i \beta z}+e^{-i \beta z}\right)\left\{\frac{\tan ^{2} \theta}{y}+b_{1}\left(1+\tan ^{2} \theta\right)\right. \\
& \left.+\left[\alpha^{2}\left(1+\frac{1}{2} \tan ^{2} \theta\right)+\frac{U_{C}^{\prime}+}{U_{C}^{\prime}}\left(1+\frac{5}{6} \tan ^{2} \theta\right)\right] y\right\} \operatorname{Re} A^{\dagger} e^{i \alpha \zeta} \\
& +\operatorname{ReF}_{1 \pm}^{(0)}\left(0, x_{1}\right) e^{2 i \beta z}+. . \\
& v_{1}=-\bar{\alpha}\left(e^{i \beta z}+e^{-i \beta z}\right) \operatorname{Re} i A^{\dagger} e^{i \alpha \zeta}+. . . \\
& w_{1}=-2 \sin \theta \sin \beta z\left[\frac{1}{y}-\frac{1}{2}\left(\bar{\alpha}^{-2}+\frac{1}{3} \frac{U_{C}^{\prime \prime}}{U_{C}^{\prime}}\right) y\right] \operatorname{Re} i A^{\dagger} e^{i \alpha \zeta}+\ldots
\end{aligned}
$$

as $y>0$, where we have anticipated the fact that the critical layer solution produces an $O(\varepsilon)$ mean flow change across the critical layer but no change in the second harmonic of the streamwise velocity fluctuation.

It follows from (2.27), (2.32), and (2.33) that there exist two continuous functions, say $\Phi_{P, 1}$ and $\Phi_{P, 2}$, which satisfy

$$
L^{(1)} \Phi_{P, 1}=\frac{U^{\prime \prime}}{U^{2}} \Phi_{1}^{(1)}
$$

and

$$
L^{(1)} \Phi_{P, 2}=p_{1}^{(1)}
$$

but are, in general, unbounded at $y= \pm \infty$. DP, 1 will behave like $\tilde{e}_{1}+\tilde{e}_{2, L y} \ln |y|+\tilde{e}_{2 y}+\ldots$, as $y \rightarrow 0$ and $\Phi p, 2$ will be regular there. The relevant solution to (2.27) must then be of the form 


$$
\begin{aligned}
& \Phi_{2}^{(1)=}=\frac{i}{\alpha}\left(U_{c} A_{x_{1}}^{\dagger}-i S_{1} A^{\dagger}\right)\left(\Phi_{P, 1}-c_{2,1}^{ \pm} \tilde{\Phi}^{(1)}-b_{2,1}^{ \pm} \tilde{\Phi}^{(2)}\right) \\
&-2 i \alpha A_{x_{1}}^{\dagger}\left(\Phi_{P, 2}-c_{2,2}^{ \pm} \tilde{\Phi}^{(1)}-b_{2,2}^{ \pm} \tilde{\Phi}^{(2)}\right)
\end{aligned}
$$

where $b_{2}^{ \pm}, n, c_{2}^{\frac{ \pm}{2}}, n$ are real constants (even on the slow scale $x_{1}$ ), which must, in general, be determined numerically.

It now follows from (2.30) that

$$
\begin{aligned}
& \bar{F}_{2}^{(1)}=-\frac{\sin ^{2} \theta}{i \alpha y} A_{x_{1}}^{\dagger}+e_{1}+e_{2} \ln |y|+2 i \alpha A_{x_{1}}^{\dagger} b_{2,2}^{ \pm} \\
& -\frac{i}{\alpha}\left(U_{c} A_{x_{1}}^{\dagger}-i S_{1} A^{\dagger}\right) b_{2,1}^{ \pm}+\ldots \text { as } y \rightarrow \pm 0
\end{aligned}
$$

where the specific forms of $e_{1}$ and $e_{2}$ are immaterial, and we do not need the corresponding expressions for $\mathrm{H}_{2}^{(1)}$ in the following analysis.

\section{THE CRITICAL LAYER}

\section{Equations (2.35) and (2.37) clearly show that the outer expansion (2.8)} to (2.11) becomes singular at the critical level. The linear small growth rate critical layer thickness is of the order of the linear growth rate which is $O\left(\varepsilon^{1 / 3}\right)$ in the present case. The appropriate scaled transverse coordinate in this region is therefore

$$
Y=\frac{y}{\varepsilon}
$$

Introducing this along with $(2.12),(2.23)$ to $(2.25),(2.40),(2.41)$, and (2.32) to (2.37) into (2.8) to (2.11) and reexpanding the result suggests that the critical layer solution should expand like

$$
\begin{gathered}
u=\varepsilon^{1 / 3} u_{c}^{\prime} Y+\varepsilon^{2 / 3} \hat{u}_{0}+\varepsilon \hat{u}_{1}+\varepsilon^{4 / 3} \hat{u}_{2}+\ldots \\
\bar{v}=-\varepsilon^{2 / 3}-\left(e^{i \beta z}+e^{-i \beta z}\right) \operatorname{Re} i A^{\dagger} e^{i \alpha \zeta}+\varepsilon \hat{v}_{1}+\varepsilon^{4 / 3 \hat{v}_{2}}+\ldots \\
w=\varepsilon^{2 / 3} \hat{w}_{0}+\varepsilon \hat{w}_{1}+\varepsilon^{4 / 3} \hat{w}_{2}+\cdots \\
p=\varepsilon \hat{p}_{0}+\varepsilon^{4 / 3} \hat{p}_{1}+\varepsilon^{5 / 3} \hat{p}_{2}+\ldots
\end{gathered}
$$


where we suppose that the $\varepsilon^{n}$ ln $\varepsilon$ terms have been incorporated in the $\hat{u}_{0}$, $\hat{u}_{1}$, etc.,

$$
\hat{p}_{0}=U_{c}^{\prime} \cos \theta\left(e^{i \beta z}+e^{-i \beta z}\right) \operatorname{Re} A^{+} e^{i \alpha \zeta}
$$

and we have put

$$
\bar{v} \equiv \varepsilon^{-1 / 3} v
$$

for convenience.

The $\hat{u}_{n}, \hat{v}_{n}, \hat{w}_{n}$, etc. are functions of $\zeta, Y, z$, and $x_{1}$, only and are determined by the inviscid momentum and continuity equations. It follows from $(2.23),(2.25),(2.28)$, and (2.41) that matching with the external solution requires that

$$
\begin{array}{r}
\frac{\alpha \beta}{2 \pi^{2}} \int_{0}^{2 \pi / \alpha} \int_{0}^{2 \pi / \beta} e^{-i(\alpha \zeta+\beta z)} \Delta \bar{u}_{2}^{+}\left(\zeta, z, x_{1}\right) d z d \zeta=2 i \alpha A_{x_{1}}^{\dagger}\left(b_{2,2}^{+}-b_{2,2}^{-}\right) \\
-\frac{i}{\alpha}\left(U_{c} A_{x_{1}}^{\dagger}-i S_{1} A^{\dagger}\right)\left(b_{2,1}^{+}-b_{2,1}^{-}\right),
\end{array}
$$

where we have put

$$
\Delta \bar{u}_{2}^{+} \equiv \lim _{Y \rightarrow \infty}\left[\bar{u}_{2}^{+}\left(\zeta, Y, Z, x_{1}\right)-\bar{u}_{2}^{+}\left(\zeta,-Y, Z, x_{1}\right)\right]
$$

and

$$
\bar{u}_{n}^{ \pm} \equiv \hat{u}_{n} \cos \theta \pm \hat{w}_{n} \sin \theta \text { for } n=0,1,2, \ldots
$$

The requirement ( 3.8 ) merely states that the change in propagation direction velocity across the critical layer, as calculated from within, is equal to the change in that velocity as calculated from the liner external solution.

It turns out to be convenient to work in terms of the spanwise vorticity

$$
\omega \equiv \frac{\partial v}{\partial x}-\frac{\partial u}{\partial y},
$$

which expands like

$$
\omega=-U_{C}^{t}-\varepsilon^{1 / 3} \hat{u}_{O Y}-\varepsilon^{2 / 3} \hat{u}_{1 Y}+\varepsilon \omega_{2}+\cdots \cdot
$$


where

$$
\omega_{2} \equiv-\hat{u}_{2 Y}+\bar{\alpha} \alpha\left(e^{i \beta z}+e^{-i \beta z}\right) \operatorname{Re} A^{+} e^{i \alpha \zeta} .
$$

Then $\omega$ and

$$
\underline{u} \equiv\{u, \bar{v}, w\}
$$

satisfy

$$
\begin{gathered}
{\left[\left(u-\varepsilon^{1 / 3} \frac{S_{1}}{\alpha}\right) \frac{\partial}{\partial \zeta}+\bar{v} \frac{\partial}{\partial Y}+\varepsilon^{1 / 3}\left(u+u_{c}\right) \frac{\partial}{\partial x_{1}}+w \frac{\partial}{\partial z}\right] \omega=\frac{1}{\varepsilon} u^{1 / 3} u_{Z} w_{Y}+w w_{Z}} \\
-\varepsilon^{1 / 3} \bar{v}_{Z} w_{\zeta}-\varepsilon^{2 / 3} \bar{v}_{Z} w_{x_{1}} \\
{\left[\left(u-\varepsilon^{1 / 3} \frac{S_{1}}{\alpha}\right) \frac{\partial}{\partial \zeta}+\bar{v} \frac{\partial}{\partial Y}+\varepsilon^{1 / 3}\left(u+u_{c}\right) \frac{\partial}{\partial x_{1}}+w \frac{\partial}{\partial z}\right] u=} \\
-\left\{p_{\zeta}+\varepsilon^{1 / 3} p_{x_{1}}, \frac{1}{\varepsilon^{2 / 3}} p_{Y}, p_{Z}\right\}
\end{gathered}
$$

and

$$
u_{\zeta}+\bar{v}_{Y}+w_{Z}+\varepsilon^{1 / 3} u_{x_{1}}=0
$$

Substituting the expansions (3.2) to (3.6) and (3.12) into (3.15) and (3.16), we find that

$$
\begin{aligned}
& \mathscr{L}_{0} \hat{w}_{0}=-U_{c}^{\prime} \bar{\alpha} \sin \theta \cos \theta \operatorname{Re} i\left(e^{i \beta z}-e^{-i \beta z}\right) A^{\dagger} e^{i \alpha \zeta} \\
& \mathscr{L}_{0} \hat{w}_{1}=\bar{\alpha}\left(e^{i \beta z}+e^{-i \beta z}\right)\left(\operatorname{Re}_{i A}^{\dagger} e^{i \alpha \zeta}\right) \hat{w}_{0 Y}-\left(\hat{u}_{0} \frac{\partial}{\partial \zeta}+\hat{w}_{0} \frac{\partial}{\partial z}\right) \hat{w}_{0}-u_{c}^{i Y \hat{w}_{0 x_{1}}}-\hat{p}_{1 z} \\
& \mathscr{L}_{0} \hat{u}_{0}=U_{c}^{\prime} \bar{\alpha} \sin ^{2} \theta\left(e^{i \beta z}+e^{-i \beta z}\right) R_{e} i A^{\dagger} e^{i \alpha \zeta} \\
& \mathscr{L}_{0} \hat{u}_{Y Y}=\bar{\alpha}\left(e^{i \beta z}+e^{-i \beta z}\right)\left(\operatorname{Re}_{i A}{ }^{\dagger} e^{i \alpha \zeta}\right) \hat{u}_{O Y Y}+\left(\hat{u}_{0} \hat{w}_{0 Z}-\hat{u}_{0 Z} \hat{w}_{0}\right)_{Y}+u_{c}^{\prime}\left(\hat{w}_{1 Z}-\hat{u}_{0 X}, Y\right)
\end{aligned}
$$




$$
\begin{aligned}
& \mathscr{L}_{0} \hat{q}_{2}=\bar{\alpha}\left(e^{i \beta z}+e^{-i \beta z}\right)\left(\operatorname{Re}_{i A} e^{i \alpha \zeta}\right)\left(\bar{u}_{I Y Y}^{+}-\cos \theta U_{C}^{\prime \prime \prime Y}\right)-\frac{U_{C}^{\prime \prime}{ }^{\prime}}{U_{C}^{\prime}}\left(e^{i \beta z}+e^{-i \beta Z}\right) \\
& \times \operatorname{Re}\left(u_{c} A_{x_{1}}^{\dagger}-i S_{1} A^{\dagger}\right) e^{i \alpha \zeta}+\left(\bar{u}_{1} \hat{w}_{0 z}-\hat{u}_{0} \bar{u}_{1 \zeta}^{+}-\hat{w}_{0} \vec{u}_{1 z}^{+}-\hat{w}_{1} \cos \theta \hat{u}_{0 z}\right. \\
& \left.-\hat{u}_{1} \sin \theta \hat{w}_{O \zeta}-\hat{v}_{1} \bar{u}_{O Y}^{+}\right)_{Y}+u_{C}^{\prime}\left(\cos \hat{\theta}_{2 Z}-\sin \hat{w}_{2 \zeta}-\sin \theta \hat{w}_{1 x_{1}}-Y \bar{u}_{1 X_{1}}^{+}\right) \\
& -\left(\hat{u}_{0} \bar{u}_{O x_{1}}^{+}\right)_{Y}
\end{aligned}
$$

and

$$
\hat{v}_{1 Y}=-\hat{u}_{1 \zeta}-\hat{w}_{1 Z}-\hat{u}_{0 x_{1}}
$$

where we have put

$$
\mathscr{L}_{0} \equiv U_{c} \frac{\partial}{\partial x_{1}}+\left(U_{c}^{\prime} Y-\frac{S_{1}}{\alpha}\right) \frac{\partial}{\partial \zeta},
$$

and

$$
\begin{aligned}
\hat{q}_{2} \equiv-\omega_{2} \cos \theta+\sin \theta\left(\hat{w}_{2 \gamma}+2 \bar{\alpha} \beta \sin \beta z\right. & \left.\operatorname{Re}_{e} A^{\dagger} e^{i \alpha \zeta}\right) \\
& -\frac{U_{C}^{\prime \prime}}{U_{C}^{\prime}}\left(e^{i \beta z}+e^{-i \beta z}\right) \operatorname{Re} A^{\dagger} e^{i \alpha \zeta}
\end{aligned}
$$

and matching with the outer solution shows that $\bar{q}_{2}$ and $\bar{u}_{1 Y}-1 / 2 U_{C}^{\prime \prime}{ }^{\prime} \cos \theta Y^{2}$ go to zero as $Y \rightarrow \pm^{\infty}$.

It is now convenient to introduce the following normalized variables

$$
\begin{gathered}
\bar{x} \equiv-\frac{1}{2} S_{1} U_{C}^{\prime} x_{1}-x_{0}, \\
n \equiv-2 \alpha\left(Y-\frac{S_{1}}{\alpha U_{C}^{\prime}}\right) / S_{1} U_{C}, \\
X \equiv \alpha \zeta-X_{0},
\end{gathered}
$$


and

$$
A \equiv 4 A^{\dagger} \alpha^{2} e^{i X_{0}}\left(S_{1} U_{C}\right)^{2} U_{C}^{\prime}
$$

where $x_{0}$ and $x_{0}$ are real constants.

Then (3.18) and (3.20) can be integrated immediately to obtain

$$
\begin{gathered}
\hat{u}_{0}=-\frac{U_{c} U_{c}^{\prime S} S_{1}}{\alpha} \sin \theta \tan \theta \cos \beta z R_{e} i w_{1}^{(0)} e^{i x} . \\
\hat{w}_{0}=-\frac{U_{c} U_{c}^{\prime S} 1}{\alpha} \sin \theta \sin \beta z \operatorname{Re}_{e} w_{1}^{(0)} e^{i x},
\end{gathered}
$$

where we have put

$$
w_{1}^{(0)}=\int_{-\infty}^{\bar{x}} e^{-i n(\bar{x}-\tilde{x})} A(\tilde{x}) d \tilde{x}
$$

It is worth noticing that

$$
W_{1}^{(0)} \rightarrow\left(\frac{i}{n-i \bar{\kappa}}\right) e^{\bar{\kappa} \bar{x}}
$$

when

$$
A \rightarrow e^{\bar{k} \bar{x}}
$$

so that (3.29) approaches the linear critical solution (given by the generalization of eq. (4.34) in Goldstein and Leib 1988) when Af approaches the linear upstream condition (2.14).

Equations (3.19) and (3.21) clearly possess solutions of the form

$$
\hat{u}_{1 Y}=\frac{1}{2} U_{C}^{\prime}{ }^{\prime} Y^{2}-\frac{1}{2} \sin \theta U_{c}^{\prime} \sum_{n, m=-2}^{2} Q_{n, m}^{(1)}(n, \bar{x}) e^{i(n X+m \beta z)} \text {, }
$$

and

$$
\hat{w}_{1}=\frac{1}{4 \alpha} s_{1} U_{c} U_{c}^{\prime} \sin \theta \sum_{n, m=-2}^{2} w_{n, m}^{(1)}(n, \bar{x}) e^{i(n x+m \beta z)} .
$$

where the first term in (3.32) was inserted to insure that $Q_{n, m}^{(1)} \rightarrow 0$ as
$n \rightarrow \pm \infty$ for all $n, m$.

Substituting (3.32) and (3.33) along with (3.29) and (3.30) into (3.19) and (3.21) shows that 


$$
\begin{gathered}
L_{0} W_{0,2}^{(1)}=i \sec \theta\left(2 \sin ^{2} \theta\left|W_{1}^{(0)}\right|^{2}-2 m A^{*} W_{1 n}^{(0)}\right), \\
L_{2} W_{2,0}^{(1)}=0 \\
L_{2} Q_{2,0}^{(1)}=\tan \theta \sec \theta\left(A W_{1 n n}^{(0)}-4 i \sin ^{2} \theta W_{1}^{(0)} W_{l n}^{(0)}\right)
\end{gathered}
$$

where the star denotes the complex conjugate, we have put

$$
L_{n} \equiv \frac{\partial}{\partial \bar{x}}+i n n \text { for } n=0,1,2, \ldots .
$$

and in writing down (3.36) we have anticipated the fact that the relevant solution to $(3.35)$ is the trivial solution

$$
w_{2,0}^{(1)}=0
$$

Inserting (3.31) into (3.34) and (3.36) and integrating yield

$$
w_{0,2}^{(1)}=i \sec \theta \operatorname{Re} \int_{-\infty}^{\bar{x}} A^{*}(\tilde{x}) e^{-i n \tilde{x}}\left[I_{1}(\tilde{x})+4 \sin ^{2} \theta(\bar{x}-\tilde{x}) I_{0}(\tilde{x})\right] d \tilde{x}
$$

and

$$
Q_{2,0}^{(1)}=-\tan \theta \sec \theta e^{-2 i n \bar{x}}\left[\int_{-\infty}^{\bar{x}} A(\tilde{x}) e^{i n \tilde{x}} I_{2}(\tilde{x}) d \tilde{x}+2 \sin ^{2} \theta I_{1}^{2}(\bar{x})\right]
$$

where we have put

$$
I_{n}(x) \equiv \int_{-\infty}^{x} e^{i n \tilde{x}}(x-\tilde{x})^{n} A(\tilde{x}) d \tilde{x} \text { for } n=0,1,2, \ldots .
$$

Inserting (3.39) along with (3.29) to (3.33) into (3.21) and integrating we obtain

$$
\begin{array}{r}
Q_{0,2}^{(1)}=\tan \theta \sec \theta \operatorname{Re} \int_{-\infty}^{\bar{x}} A^{*}(\tilde{x}) e^{-i n \tilde{x}}\left[I_{2}(\tilde{x})-2(\bar{x}-\tilde{x}) I_{1}(\tilde{x})\right. \\
\left.-4(\bar{x}-\tilde{x})^{2} \sin ^{2} \theta I_{0}(\tilde{x})\right] d \tilde{x} .
\end{array}
$$

Since $\hat{u}_{1 y}$ and $\hat{w}_{1}$ are real we must set

$$
w_{0,-2}^{(1)}=w_{0,2}^{(1)^{*}}
$$




$$
\begin{gathered}
Q_{0,-2}^{(1)}=Q_{0,2}^{(1)^{*}} \\
W_{-2,0}^{(1)}=W_{2,0}^{(1)^{*}}=0
\end{gathered}
$$

and

$$
Q_{-2,0}^{(1)}=Q_{2,0}^{(1)^{*}}
$$

It is easy to show that

$$
\begin{gathered}
Q_{0,0}^{(1)} \cos \theta+W_{0,0 n}^{(1)} \sin \theta=\tan \theta \operatorname{Re} \int_{-\infty}^{\bar{x}} A^{*}(\tilde{x}) e^{-i n \tilde{x}} I_{2}(\tilde{x}) d \tilde{x} \\
Q_{1,1}^{(1)}=\frac{1}{2 \alpha} S_{1} U_{c} \sin \theta W_{\ln \bar{x}}^{(0)}
\end{gathered}
$$

and

$$
\begin{aligned}
{\left[W_{1,1}^{(1)} \sin \theta-\frac{2 \alpha Y}{S_{1} U_{c}}\left(Q_{1,1}^{(1)} \cos \theta+W_{1, \ln }^{(1)} \sin \theta\right)\right]_{\bar{x}} } & \\
& =\hat{C}_{1}\left(W_{1,1}^{(1)}-\frac{i}{2 \alpha} s_{1} U_{c}^{\prime} W_{1 \bar{x}}^{(0)}\right)_{n}
\end{aligned}
$$

where $\hat{C}$ is a constant whose specific value will not effect our analysis. The remaining $Q$ 's and $W^{\prime} s$ can be similarly determined, but are not used in the following analysis. the form

It now follows from (3.32) and (3.33) that (3.22) possesses a solution of

$$
\hat{q}_{2}=-\frac{1}{2} \sin \theta U_{c}^{\prime} \sum_{n, m=-3}^{3} Q_{n, m}^{(2)}(n, \bar{x}) e^{i(n X+m \beta z)} .
$$

where

$$
Q_{n, m}^{(2)} \rightarrow 0 \text { as } n \rightarrow \pm \infty \text {. }
$$

Substituting this along with (3.32), (3.33), (3.48), (3.38), and (3.43) to (3.46) into (3.22) and (3.23) yields 


$$
\begin{aligned}
& L_{1}\left[Q_{1,1}^{(2)}-\hat{c}\left(w_{1,1}^{(1)}-\frac{i}{2 \alpha} s_{1} U_{c}^{\prime} w_{1}^{(0)}\right)_{n}\right]=\frac{i}{2}\left\{A\left[Q_{0,2}^{(1)}+Q_{0,0}^{(1)}+\tan \theta\left(w_{0,2}^{(1)}+w_{0,0}^{(1)}\right)_{n}\right]_{n}\right. \\
& \left.-A^{*} Q_{2,0 n}^{(1)}\right\}+2 \sin ^{2} \theta \frac{\partial}{\partial \eta}\left[w_{1}^{(0)} U_{0,2}^{(1)}-w_{\ln }^{(0)} v_{0,2}^{(1)}+w_{\ln }^{(0) *} v_{2,0}^{(1)}\right] \\
& +\frac{U_{C} S_{l}^{2}}{2 \alpha^{2} \sin \theta}\left(\frac{U_{C}^{\prime \prime}}{U_{C}^{\prime}{ }^{2}}\right)\left(\frac{1}{2} U_{C} U_{C}^{\prime} A_{\bar{x}}+i A\right)
\end{aligned}
$$

where

$$
\begin{gathered}
u_{0,2 n}^{(1)}=Q_{0,2}^{(1)}, \\
v_{0,2 n}^{(1)}=\tan \theta w_{0,2}^{(1)},
\end{gathered}
$$

and

$$
v_{2, O n n}^{(1)}=Q_{2,0}^{(1)}
$$

Inserting (3.39) and (3.42), we find that

$$
\begin{array}{r}
R_{0,2} \equiv 2 \sin ^{2} \theta \frac{\partial}{\partial \eta}\left(w_{1}^{(0)} u_{0,2}^{(1)}-w_{1 n}^{(0)} v_{0,2}^{(1)}\right)=\sin \theta \tan ^{2} \theta\left(w_{1}^{(0)} J_{3 \bar{x}}+2 w_{1 n}^{(0)} J_{2 \bar{x} \bar{x}}\right. \\
\left.-w_{1 \eta \eta}^{(0)} J_{1 \bar{x} \bar{x} \bar{x}}\right)
\end{array}
$$

where the $J_{n}$ 's depends on both $\bar{x}$ and $n$ and are defined by

$$
\begin{gathered}
J_{I \eta}=\frac{i}{3} \operatorname{Re} \int_{-\infty}^{\bar{x}} A^{*}(\tilde{x})(\bar{x}-\tilde{x})^{3}\left[(\bar{x}-\tilde{x}) \sin ^{2} \theta+i \frac{\partial}{\partial \eta}\right] e^{-i n \tilde{x}} I_{0}(\tilde{x}) d \tilde{x} \\
J_{2}=i\left(J_{1}-J_{4}\right)
\end{gathered}
$$

where

$J_{4} \equiv \frac{2}{3} \sin ^{2} \theta \operatorname{Re} \int_{-\infty}^{\bar{x}} A^{*}(\tilde{x})(\bar{x}-\tilde{x})^{3} e^{-i n \tilde{x}} I_{0}(\tilde{x}) d \tilde{x}+\frac{1}{2} \int_{-\infty}^{\bar{x}} A(\tilde{x})(\bar{x}-\tilde{x})^{2} e^{i n \tilde{x}} I_{1}^{*}(\tilde{x}) d \tilde{x}$ 
and

$$
J_{3}=-2 \operatorname{Re} \int_{-\infty}^{\bar{x}} A^{*}(\tilde{x})(\bar{x}-\tilde{x})\left[\frac{4}{3} \sin ^{2} \theta(\bar{x}-\tilde{x})^{2} I_{0}(\tilde{x})+(\bar{x}-\tilde{x}) I_{1}(\tilde{x})-I_{2}(\tilde{x})\right] e^{-i n \tilde{x}} d \tilde{x}
$$

Then, upon integrating by parts and inserting (3.31), we obtain

$$
\begin{aligned}
\int_{-\infty}^{\bar{x}} e^{-\ln (\bar{x}-\tilde{x})} R_{0,2} d \tilde{x} & =\sin \theta \tan ^{2} \theta e^{-i n \bar{x}}\left\{J_{1 \bar{x} \bar{x}} I_{2}(\bar{x})-2 J_{4 \bar{x}} I_{1}(\bar{x})\right. \\
& \left.-\int_{-\infty}^{\bar{x}} e^{i n \tilde{x}} A(\tilde{x})\left[2 J_{4}(\tilde{x})-2 J_{4}(\bar{x})+J_{3}(\tilde{x})-J_{3}(\bar{x})\right] d \tilde{x}\right\} .
\end{aligned}
$$

Substituting (3.40) into (3.55) we find after some manipulation that

$$
\begin{aligned}
V_{2,0}^{(1)}=-\frac{1}{2} e^{-2 \operatorname{in} \bar{x}}\left(\frac{\partial}{\partial \bar{x}} e^{2 i n \bar{x}_{U}(1)}{ }_{2,0}\right)+\frac{1}{2} \tan \theta \sec \theta\left[A(\bar{x}) e^{-i n \bar{x}} I_{1}(\bar{x})\right. \\
\left.+2 \sin ^{2} \theta e^{-2 i n \bar{x}} I_{0}^{2}(\bar{x})\right],
\end{aligned}
$$

where

$$
u_{2, O n}^{(1)}=Q_{2,0}^{(1)}
$$

Inserting this along with (3.31) into

$$
\int_{-\infty}^{\bar{x}} e^{-i \eta(\bar{x}-\tilde{x})} R_{2,0} d \tilde{x}
$$

where

$$
R_{2,0} \equiv 2 \sin ^{2} \theta \frac{\partial}{\partial n} w_{l n}^{(0) *} v_{2,0}^{(1)}
$$

and integrating by parts now shows that

$$
\begin{aligned}
& \int_{-\infty}^{\bar{x}} e^{-i n(\bar{x}-\tilde{x})} R_{2,0} d \tilde{x}=i \sin ^{2} \theta e^{i n \bar{x}} I_{2}^{*}(\bar{x}) U_{2,0}^{(1)}(\bar{x}) \\
& \quad-\sin \theta \tan ^{2} \theta e^{-i n \bar{x}} \int_{-\infty}^{\bar{x}} I_{2}^{*}(\tilde{x})\left[A(\tilde{x}) e^{i n \tilde{x}} I_{1}(\tilde{x})+2 \sin ^{2} \theta I_{0}^{2}(\tilde{x})\right] d \tilde{x} .
\end{aligned}
$$


It is now easy to write down the solution to (3.52) by using (3.61), (3.65), $(3.39),(3.42)$, and $(3.47)$. Using the resulting formula for $Q(2), 1$ and integrating by parts shows that

$$
\begin{aligned}
\frac{1}{2 \pi} \int_{-\infty}^{\infty} Q_{1,1}^{(2)} d n & =\frac{U_{c} S_{1}^{2} U_{C}^{\prime \prime}}{4 \alpha^{2} \sin \theta U_{c}^{\prime 2}}\left(\frac{1}{2} U_{c} U_{c}^{\prime} A_{\bar{x}}+i A\right) \\
& +\sec \theta \tan \theta \int_{-\infty}^{\bar{x}} \int_{-\infty}^{\bar{x}} K\left(\bar{x} \mid \tilde{x}_{,} \tilde{x}_{1}\right) A(\tilde{x}) A\left(\tilde{x}_{1}\right) A\left(\tilde{x}+\tilde{x}_{1}-\bar{x}\right) d \tilde{x}, d \tilde{x},
\end{aligned}
$$

where we have put

$$
\begin{aligned}
K\left(\bar{x} \mid \tilde{x}, \tilde{x}_{1}\right) \equiv(\bar{x}-\tilde{x})\left\{\left[\frac{1}{2}(\bar{x}-\tilde{x})\right.\right. & \left.+\sin ^{2} \theta\left(\tilde{x}-\tilde{x}_{1}\right)\right]\left[2\left(\tilde{x}-\tilde{x}_{1}\right)-(\bar{x}-\tilde{x})\right] \\
& \left.-4 \sin ^{4} \theta\left[\left(\tilde{x}-\tilde{x}_{1}\right)^{2}+(\bar{x}-\tilde{x})\left(\bar{x}-\tilde{x}_{1}\right)\right]\right\} .
\end{aligned}
$$

It now follows from (3.8) and (3.24) to (3.28) that

$\sin \theta \int_{-\infty}^{\infty} Q_{1,1}^{(2)} d \eta=\frac{1}{2} i s_{1}^{2} U_{c}\left[U_{c}^{\prime} A_{\bar{x}}\left(b_{2,2}^{+}-b_{2,2}^{-}\right)-\frac{1}{\alpha}\left(\frac{U_{c} U_{C}^{\prime}}{2} A_{\bar{x}}+i A^{\prime}\right)\left(b_{2,1}^{+}-b_{2,1}^{-}\right)\right]$.

Inserting ( 3.66 ) into this result yields

$$
\frac{1}{\bar{k}} A_{\bar{x}}=A+y \tan ^{2} \theta \int_{-\infty}^{\bar{x}} \int_{-\infty}^{\tilde{x}} K\left(\bar{x} \mid \tilde{x}, \tilde{x}_{1}\right) A(\tilde{x}) A\left(\tilde{x}_{1}\right) A^{*}\left(\tilde{x}+\tilde{x}_{1}-\bar{x}\right) d \tilde{x} 1 d \tilde{x},
$$

where

$$
\frac{1}{\kappa}=\gamma S_{1}^{2} U_{c} \frac{i}{4 \pi} U_{c}^{\prime}\left(b_{2,2}^{+}-b_{2,2}^{-}\right)+\frac{i U_{c} U_{c}^{\prime}}{2}
$$

is the reciprocal of the scaled complex wave number in the linear amplitude (2.14) and we have put

$$
\frac{1}{\gamma}=-\frac{1}{\pi}\left(b_{2,1}^{+}-b_{2,1}^{-}+\frac{i \pi U_{C}^{\prime \prime}}{U_{C}^{\prime 2}}\right) \frac{s_{1}^{2} U_{C}}{4 \alpha^{2}}
$$

Equation (3.69) is the final result. It determines the amplitude of the instability wave. It must be solved subject to the upstream boundary condition 


$$
A \rightarrow e^{\bar{k} \bar{x}+i \varphi_{0}} \text { as } \bar{x} \rightarrow-\infty,
$$

where the real constant $\varphi_{0}$ is an as yet unspecified initial phase factor which was introduced via the arbitrary origin shifts $x_{0}$ and $x_{0}$ in (3.25) and (3.27).

\section{ASYMPTOTIC SOLUTION OF AMPLITUDE EQUATION}

The principal result of this paper is given by (3.69) together with the upstream boundary condition (3.72). The numerical solution to this problem, which is discussed in the next section, appears to develop a singularity at a finite value of $\bar{x}_{\text {, say }} \bar{x}_{s}$. In this section we determine the asymptotic form of the solution as $\bar{x} \rightarrow \bar{x}_{S}$. To this end we substitute

$$
A=\frac{a}{\left(\bar{x}_{s}-\bar{x}\right)^{3+i \sigma}} \text {, }
$$

where $\bar{x}_{5}$ and $\sigma$ are real constants and a is a complex constant, into the integral of (3.69) and change the integration variables from $\tilde{x}_{1}$ and $\tilde{x}$ to $\left(\bar{x}_{S}-\bar{x}_{1}\right) /\left(\bar{x}_{S}-\bar{x}\right)$ and $\left(\bar{x}_{S}-\bar{x}\right) /\left(\bar{x}_{S}-\bar{x}\right)$ to show that

$$
\int_{-\infty}^{\bar{x}} \int_{-\infty}^{\tilde{x}} K\left(\bar{x} \mid \tilde{x}, \tilde{x}_{1}\right) A(\tilde{x}) A\left(\tilde{x}_{1}\right) A^{*}\left(\tilde{x}+\tilde{x}_{1}-\bar{x}\right) d \tilde{x}_{1} d \tilde{x}=\frac{a|a|^{2}}{\left(\bar{x}_{5}-\bar{x}\right)^{4+\tilde{1} \sigma}} D(\sigma) \text {, }
$$

where

$$
\begin{aligned}
D(\sigma)= & \int_{1}^{\infty} \frac{1}{v^{3+i \sigma}} \int_{v}^{\infty} \frac{d u d v}{u^{3+i \sigma}(u+v-1)^{3-i \sigma}} \\
& \times\left[-\frac{1}{2}(v-1)^{3}+(v-1)^{2}\left\{u-v-\sin ^{2} \theta\left[u-v+4(u-1) \sin ^{2} \theta\right]\right\}\right. \\
& \left.+2(v-1)(u-v)^{2} \sin ^{2} \theta\left(1-2 \sin ^{2} \theta\right)\right] \\
& =\int_{1}^{\infty} \frac{1}{v^{3+i \sigma}(v-1)^{2}}\left\{\sum_{m=-2}^{2} \frac{\hat{c}_{m}(v \mid \theta)}{m+i \sigma}\left[\left(\frac{2 v-1}{v}\right)^{m+i \sigma}-1\right]\right) d v \\
& =\int_{0}^{1} \frac{(1-x)^{3+i \sigma}}{x^{2}}\left\{\sum_{m=-2}^{2} \frac{\tilde{c}_{m}(x \mid \theta)}{m+i \sigma}\left[\left(\frac{2 v-1}{v}\right)^{m+i \sigma}-1\right]\right\} d x
\end{aligned}
$$

and the $\hat{c}_{m}$ and $\tilde{c}_{m}$ are complicated functions of the indicated arguments which are listed in the appendix. It is worth noting that $\hat{c}_{m}$ is a polynomial 
in $\sin \theta$ whereas $\tilde{C}_{m}$ is a Fourier series in $\theta$. Carrying out the last integration gives

$$
\begin{aligned}
& D(\sigma)=\sum_{n=1}^{\infty}(-1)^{n}\left[\frac{c_{1,0}(n \mid \sigma)+c_{1,2}(n \mid \sigma) \cos 2 \theta+C_{1,4}(n \mid \sigma) \cos 4 \theta}{n(n+1)(n+2)(n+3)(4+i \sigma)} n\right. \\
& +\frac{C_{2,0}(n \mid \sigma)+C_{2,2}(n \mid \sigma) \cos 2 \theta+C_{2,4}(n \mid \sigma) \cos 4 \theta}{(n+1)(n+2)(n+3)(4+i \sigma) n} \\
& \left.+\frac{c_{3,0}(n \mid \sigma)+C_{3,2}(n \mid \sigma) \cos 2 \theta+C_{3,4}(n \mid \sigma) \cos 4 \theta}{(n+2)(n+3)(4+i \sigma) n}\right],
\end{aligned}
$$

where $(\alpha)_{n}$ denotes the generalized factorial function $\Gamma(\alpha+n) / \Gamma(\alpha)$ and the coefficients $C_{1,0} \ldots \ldots, C_{3,4}$ are given in the appendix.

$A \bar{x}$ becomes large compared to $A$ as $\bar{x} \rightarrow \bar{x}_{S}$, and the left hand side of (3.69) is balanced by the integral term on the right side. Substituting (4.1) into the left hand side shows that the two terms will balance when $\sigma$ satisfies

$$
\frac{D(\sigma)}{(3+\dot{i \sigma)}} \tan ^{2} \theta=\frac{1}{\gamma \bar{\kappa}|a|^{2}}
$$

Figures 1 and 2 show $\sigma$ and $|a \sqrt{\gamma \bar{k}}|$ respectively as functions of arg $(1 / \gamma \bar{K})$, which are used to evaluate asymptotic curves in figures 3 to 6 (shown as dotted lines).

Parts of the derivations in this section were done with MACSYMA (412.61 for DEC VAX 8600 Series) batch programs. Interested readers can contact the second author for the programs.

\section{NUMERICAL RESULTS AND DISCUSSION}

The relevant solutions to (3.69) and (3.72) involve the two complex parameters $\vec{k}$ and $y$, the propagation angle $\theta$, and, the still unspecified initial phase factor $\varphi_{0}$. But introducing the rescaled variables $A /|Y|^{1 / 2}|\bar{\kappa}|^{2}$ and $|\bar{x}| \bar{x}-\bar{x}_{0}$, where $\bar{x}_{0}$ and $\varphi_{O}$ are chosen so that

$$
\frac{\bar{\kappa}}{|\bar{\kappa}|} \bar{x}_{0}+i \varphi_{0}=\ln |y|^{1 / 2}|\bar{\kappa}|^{2} \text {, }
$$

shows that these solutions can be completely characterized by the two imaginary parameters $\ln (\bar{k} /|\bar{k}|)$ and $\ln (y /|y|)$, or equivalently by the arguments of $\bar{k}$ and $y$. The real part of $\bar{k}$ is the scaled growth rate of the upstream linear instability wave in the vicinity of its neutral stability point, and the imaginary part is the scaled deviation of the wave number from its neutral value corresponding to the prescribed strouhal number deviation $S_{1}$.

Equation (3.69) was solved numerically by using a fourth order predictor corrector scheme to advance the solution downstream from the prescribed 
upstream linear state (eq. (3.72)). The double integrals were computed by using the Trapezoidal rule with the upstream "tails" evaluated analytically from the upstream linear solutions. $\bar{\kappa}$ and $\gamma$ must, in general, be found numerically by solving the homogeneous and inhomogeneous Rayleigh's equations (2.19) and (2.27). Analytic solutions can only be obtained for the "tanh" shear layer (Huerre, 1980; 1987) where

$$
\begin{array}{ll}
U_{C}=\left(U^{(1)}+U^{(2)}\right) / 2 \Delta, \quad U_{C}^{\prime}=\bar{\alpha}=1, \quad U_{C}^{\prime \prime}=-2, & b_{2,1}^{+} \quad J_{2,1}^{-}=0, \\
& \text { and } b_{2,2}^{+}-b_{2,2}^{-}=1 .
\end{array}
$$

Figures 3 and 5 are plots of the instability wave growth rate $|A| \bar{x} /|A|$ for various values arg $\gamma$, arg $\bar{k}$, and $\cos \theta$. We only show results for $-\pi / 2<\arg \bar{\kappa}<0$ because (3.69) implies that $A\left(\bar{x}, \bar{\kappa}^{\star}, \gamma^{\star}\right)=A^{\star}(\bar{x}, \bar{\kappa}, \gamma)$. Notice that the upstream linear growth rate is initially reduced when arg $Y$ is in the range $-\pi / 2<\arg \bar{\kappa}_{Y}<\pi / 2$. This is because the nonlinear term behaves like $-\gamma C_{O}|A|^{2} A$ for small $|A|$, where $C_{O}$ is a positive real constant. The effective growth rate is therefore reduced by the factor $1-C_{0}|A|^{2} \gamma$, but (except in the special case arg $Y=\arg \bar{\kappa}=0$ ) this trend is eventually reversed, and the yiuwth rate rapidly increases until the amplitude becomes sillgular at some finite downstream distance for all values of $Y$ - suggesting an explosive growth of the instability wave there.

This is shown somewhat better in figures 6 to 8 , which are plots of the real part of the scaled instability wave amplitude versus the scaled streamwise coordinate $\bar{x}$. Also shown in the figures are the results computed from the asymptotic solution ( 4.1 ), with the singularity location $\bar{x}_{5}$ determined from the numerical solution. The latter solutions clearly approach the asymptotic result as $\left|\bar{x}_{S}-\bar{x}\right|$ becomes small. Since $(4,1)$ implies that the asymptotic growth rate $|A| \bar{x} /|A|$ behaves like $(|A| /|a|) i / 3$ in the vicinity of the singularity, the initial scaling, i.e., growth rate $=O(\varepsilon)^{1 / 3}$, is unchanged by the singularity. This suggests that the basic asymptotic structure of the critical layer will remain intact, and the present solution will not break down until the amplitude $|A|$ of the external instability wave becomes order one. The flow will then be fully nonlinear and unsteady in the main part of the shear layer, i.e., it will be governed by the full Euler's equations there.

This is quite different from the nonlinear critical layer behavior found by Goldstein and Leib (1988) for the two-dimensional case where the critical layer nonlinearity produced a redistribution of vorticity that rapidly reduced the instability wave growth rate to zero. The three-dimensional effects in the present analysis allow for vortex stretching, which completely counteracts this effect and produces the explosive instability wave growth. This phenomena is probably masked at subsonic speeds by the fact that the linear growth rate of the three-dimensional wave is much smaller than that of the two-dimensional wave. The latter wave can alter the critical-layer structure of the oblique waves, and the present analysis assumes that the two-dimensional wave is completely absent in the nonlinear region. It should be possible to eliminate the two-dimensional wave in carefully controlled subsonic experiments, but it would probably be much easier to observe the phenomena at supersonic speeds where the most rapidly growing linear mode is oblique. While the basic amplitude equation (3.69) was derived only for incompressible flow, it applies to the compressible case as well, and there is no a-priori restriction on the Mach number. It is worth noting that the nonlinear critical layer effects 
should be much more important at higher Mach numbers, because the linear growth rate rapidly decreases with increasing Mach number (Jackson and Grosch, 1988).

Figures 6 to 8 show that the instability wave amplitude undergoes successive oscillation upstream of the singularity. Similar behavior was observed in the two-dimensional analysis of Goldstein and Leib (1988) and in the calculations of Benney and Maslowe (1975), Huerre (1977), and Miura and Sato (1978). The amplitude oscillations imply periodic reversal of energy transfer between the fluctuations and the mean flow, and possibly between the fluctuations themselves. By considering the Reynolds-stress changes that occur with nutating elliptic vortices, Browand and Ho (1983) came up with a simple kinematic explanation for this phenomenon. The reader is referred to Ho and Huerre (1984, p. 410) for details.

Viscous effects will undoubtedly alter the critical layer behavior described above. We decided not to include them because they would greatly complicate an already complex analysis. They were, however, included in the simpler analysis of Goldstein and Leib (1989), and we expect them to act similarly in the present situation. The former analysis suggests that viscous effects always delay the explosive growth and that they will eliminate it entirely for a certain range of values of $\gamma, \bar{\kappa}$, and $\theta$, provided an appropriate scaled viscous parameter exceeds a certain finite value. In that case, the solution will just go to a finite amplitude equilibrium state further downstream.

While the present scaling may seem to be rather special, the composite expansion technique of Goldstein and Leib (1988) shows that the instability wave adjusts to the proper scaling automatically as it propagates downstream toward the neutral stability point. The nonlinear region actually sets its own location in the final composite expansion. The only requirement is that the instability wave amplitude remain fairly small in the region where the linear growth rate is fairly small. This requirement should be very nonrestrictive at supersonic speeds where the linear growth rate is always small. However, the experiments show that nonlinearity sets in at very small amplitudes, even at subsonic speeds, which suggests that the nonlinear effects are localized and therefore confined to the critical layer (since this is the region where nonlinearity would first come into play; Goldstein and Leib, 1988; Goldstein and Hultgren, 1988). when

Equations (2.7), (3.25), and (4.1) show that the explosive growth occurs

$$
x-x_{s}=0(1)
$$

where

$$
x_{S} \equiv \frac{\bar{x}_{S}+\bar{x}_{O}}{-\frac{1}{2} \varepsilon^{1 / 3} S_{1} U_{C}^{\prime}}
$$

IAs in Goldstein and Leib (1988), the nonlinear solution can be considered to be an inner solution in a composite expansion whose outer solution is the linear wave. 
is the singularity location in the unscaled streamwise coordinates. The streamwise extent of the fully nonlinear region is therefore of the same order as the shear layer width.

It is also worth noting that the asymptotic instability wave amplitude is uniquely determined by the asymptotic solution and is therefore independent of the upstream conditions. Figures 9 to 11 show the wavelength reduction $I_{m}\left(A_{\bar{x}} / A\right)$ as a function of the scaled streamwise coordinate $\bar{x}$. The asymptotic results computed from (4.1) are indicated by the dashed lines.

The authors would like to thank Dr. Lennart Hultgren for his helpful comments on the manuscript and Dr. S.J. Leib for helping with the computer program. 


\section{APPENDIX}

The detailed expressions for coefficients used in (4.3) and (4.4) are

$$
\begin{aligned}
& \hat{c}_{2}=\frac{3 v-1}{2(v-1)}-\frac{v(3 v-1)}{(v-1)^{2}} \sin ^{2} \theta+4 \frac{v^{2}-v+1}{(v-1)^{2}} \sin ^{4} \theta \text {. } \\
& \hat{c}_{1}=-\frac{7 v-3}{v-1}+\frac{17 v^{2}-10 v+1}{(v-1)^{2}} \sin ^{2} \theta-4 \frac{5 v^{2}-4 v+3}{(v-1)^{2}} \sin ^{4} \theta \text {, } \\
& \hat{c}_{0}=6 \frac{2 v-1}{v-1}-\frac{35 v^{2}-28 v+5}{(v-1)^{2}} \sin ^{2} \theta+8 \frac{5 v^{2}-4 v+2}{(v-1)^{2}} \sin ^{4} \theta \text {, } \\
& \hat{c}_{-1}=-\frac{9 v-5}{v-1}+\frac{31 v^{2}-30 v+7}{(v-1)^{2}} \sin ^{2} \theta-4 \frac{9 v^{2}-8 v+3}{(v-1)^{2}} \sin ^{4} \theta \text {, } \\
& \hat{c}_{-2}=\frac{5 v-3}{2(v-1)}-\frac{10 v^{2}-11 v+3}{(v-1)^{2}} \sin ^{2} \theta+4 \frac{3 v^{2}-3 v+1}{(v-1)^{2}} \sin ^{4} \theta \text {, } \\
& \tilde{C}_{2}=\frac{1}{2}\left(1-2 x+4 x^{2}\right)-\frac{1}{2}\left(2-5 x+4 x^{2}\right) \cos 2 \theta+\frac{1}{2}\left(1-x+x^{2}\right) \cos 4 \theta \text {, } \\
& \tilde{c}_{1}=-\left(2-3 x+7 x^{2}\right)+\frac{1}{2}\left(8-16 x+11 x^{2}\right) \cos 2 \theta-\frac{1}{2}\left(4-2 x+3 x^{2}\right) \cos 4 \theta, \\
& \tilde{c}_{0}=\frac{1}{2}\left(6-6 x+19 x^{2}\right)-\frac{1}{2}\left(12-18 x+11 x^{2}\right) \cos 2 \theta+\left(3+2 x^{2}\right) \cos 4 \theta, \\
& \tilde{c}_{-1}=-\left(2-x+6 x^{2}\right)+\frac{1}{2}\left(8-8 x+5 x^{2}\right) \cos 2 \theta-\frac{1}{2}\left(4+2 x+3 x^{2}\right) \cos 4 \theta, \\
& \tilde{c}_{-2}=\frac{1}{2}\left(1+3 x^{2}\right)-\frac{1}{2}\left(2-x+x^{2}\right) \cos 2 \theta+\frac{1}{2}\left(1+x+x^{2}\right) \cos 4 \theta \text {, } \\
& c_{1,0}=\frac{1}{2}(-1-i \sigma)_{n+2}-2(-i \sigma)_{n+2}+3(1-i \sigma)_{n+2}-2(2-i \sigma)_{n+2}+\frac{1}{2}(3-i \sigma)_{n+2} \text {, } \\
& c_{2,0}=-(-1-i \sigma)_{n+2}+3(-i \sigma)_{n+2}-3(1-i \sigma)_{n+2}+(2-i \sigma)_{n+2} \text {, } \\
& c_{3,0}=2(-1-i \sigma)_{n+2}-7(-i \sigma)_{n+2}+\frac{19}{2}(1-i \sigma)_{n+2}-6(2-i \sigma)_{n+2}+\frac{3}{2}(3-i \sigma)_{n+2} \text {, }
\end{aligned}
$$




$$
\begin{aligned}
& c_{1,2}=-(-1-i \sigma)_{n+2}+4(-i \sigma)_{n+2}-6(1-i \sigma)_{n+2}+4(2-i \sigma)_{n+2}-(3-i \sigma)_{n+2}, \\
& c_{2,2}=\frac{5}{2}(-1-i \sigma)_{n+2}-8(-i \sigma)_{n+2}+9(1-i \sigma)_{n+2}-4(2-i \sigma)_{n+2}+\frac{1}{2}(3-i \sigma)_{n+2}, \\
& c_{3,2}=-2(-1-i \sigma)_{n+2}+\frac{11}{2}(-i \sigma)_{n+2}-\frac{11}{2}(1-i \sigma)_{n+2}+\frac{5}{2}(2-i \sigma)_{n+2} \\
& c_{1,4}=\frac{1}{2}(-1-i \sigma)_{n+2}-2(-i \sigma)_{n+2}+3(1-i \sigma)_{n+2}-2(2-i \sigma)_{n+2}+\frac{1}{2}(3-i \sigma)_{n+2}, \\
& c_{2,4}=-\frac{1}{2}(-1-i \sigma)_{n+2}+(-i \sigma)_{n+2}-(2-i \sigma)_{n+2}+\frac{1}{2}(3-i \sigma)_{n+2}, \quad(A .18)_{n+2}, \\
& c_{3,4}=\frac{1}{2}(-1-i \sigma)_{n+2}-\frac{3}{2}(-i \sigma)_{n+2}+2(1-i \sigma)_{n+2}-\frac{3}{2}(2-i \sigma)_{n+2}+\frac{1}{2}(3-i \sigma)_{n+2},
\end{aligned}
$$




\section{REFERENCES}

1. Benney, D.J.; and Maslowe, S.A.: The Evolution in Space and Time of Nonlinear Waves in Parallel Shear Flows. Stud. Appl. Math., vol. 54, no. 3, Sept. 1975, pp. 181-205.

2. Browand, F.K.; and Ho, C.M.: The Mixing Layer: An Example of Quasi Two-Dimensional Turbulence. J. Mec. Theor. Appl., vol. 2, Spec. Suppl., 1983, pp. 99-120.

3. Goldstein, M.E.; and Hultgren, L.S.: Nonlinear Spatial Evolution of an Externally Excited Instability Wave in a Free Shear Layer. J. Fluid Mech., vol. 197, Dec. 1988, pp. 295-330.

4. Goldstein, M.E.; and Leib, S.J.: Nonlinear Roll-up of Externally Excited Free Shear Layers. J. Fluid Mech., vol 191, June 1988, pp. 481-515.

5. Goldstein, M.E.; and Leib, S.J.: Nonlinear Evolution of Oblique Waves on Supersonic Shear Layers. Submitted to J. Fluid Mech., 1989.

6. Gropengiesser, H.: Study on the Stability of Boundary Layers and Compressible Fluids. Deutsche Luft--und Raumfahrt, Report DLR-FB-69-25, NASA TT F-12786, 1969.

7. Hickernell, F.J.: Time-Dependent Critical Layers in Shear Flows on the Beta-Plane. J. Fluid Mech., vol. 142, May 1984, pp. 431-449.

8. Ho, C.M.; and Huerre, P.: Perturbed Free Shear Layers. Annual Review of Fluid Mechanics, Vol. 16, M. Van Dyke, J.V. Wehausen, and J.L. Lumley, eds., Annual Reviews Inc., 1984, pp. 365-424.

9. Huerre, P.: Nonlinear Instability of Free Shear Layers. LaminarTurbulent Transition, AGARD CP-224, AGARD, Paris, France, 1977, pp. 5-1 to 5-12. (Avail. NTIS, AD-A048990.)

10. Huerre, P.: The Nonlinear Stability of a Free Shear Layer in the Viscous Critical Layer Regime. Philos. Trans. R. Soc. London A, vol. 293, no. 1408, Jan. 10, 1980, pp. 643-672.

11. Huerre, P.: On the Landau Constant in Mixing Layers. Proc. R. Soc. London A, vol. 409, no. 1837, Feb. 9, 1987, pp. 369-381.

12. Huerre, P.; and Scott, J.F.: Effects of Critical Layer Structure on the Nonlinear Evolution of Waves in Free Shear Layers. Proc. R. Soc. London A, vol. 371, no. 1747, Aug. 4, 1980, pp. 509-524.

13. Jackson, T.L.; and Grosch, C.E.: Spatial Stability of a Compressible Mixing Layer. NASA CR-181671, 1988.

14. Maslowe, S.A.: Critical Layers in Shear flows. Annual Review of Fluid Mechanics, Vol. 18, M. Van Dyke, J.V. Wehausen, and J.L. Lumley, eds., Annual Reviews Inc., 1986, pp. 405-432. 
15. Miura, A.; and Sato, T.: Theory of Vortex Nutation and Amplitude Oscillation in an Inviscid Shear Instability. J. Fluid Mech., vol. 86 , pt. 1, May 15, 1978, pp. 33-47.

16. Robinson, J.L.: The Inviscid Nonlinear Instability of Parallel Shear Flows. J. Fluid Mech., vol. 63, pt. 4, May 15, 1974, pp. 723-752.

17. Stewartson, K.: Marginally Stable Inviscid Flows With Critical Layers. IMA J. App I. Math., vol. 27, no. 2, 1981, pp. 133-175.

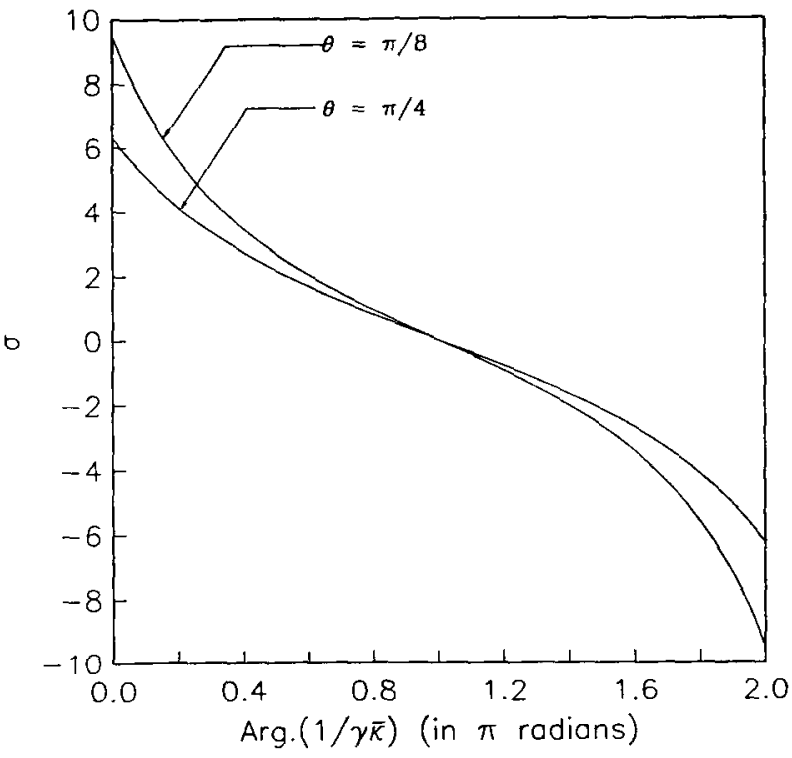

FIGURE 1. - ASYMPTOTIC EXPONENT O VERSUS ARG. $(1 / \gamma \bar{\kappa})$ IN $\pi$ RADIANS. 


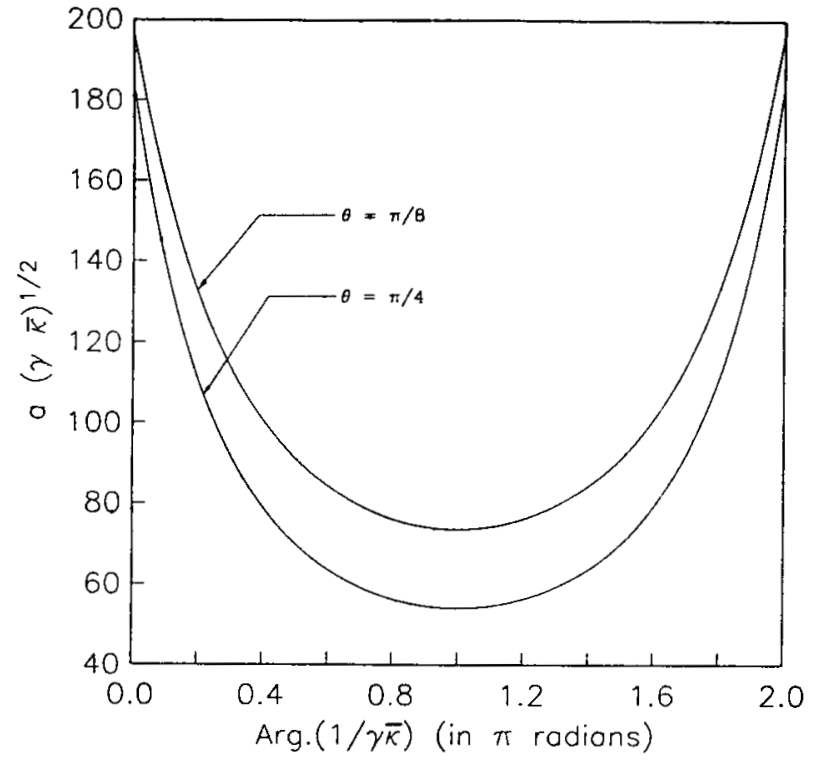

FIGURE 2. - NORMALIZED ASYMPTOTIC AMPLITUDE $a \sqrt{\gamma \bar{K}}$ VERSUS ARG. $(1 / \gamma \bar{K})$ IN $\pi$ RADIANS.

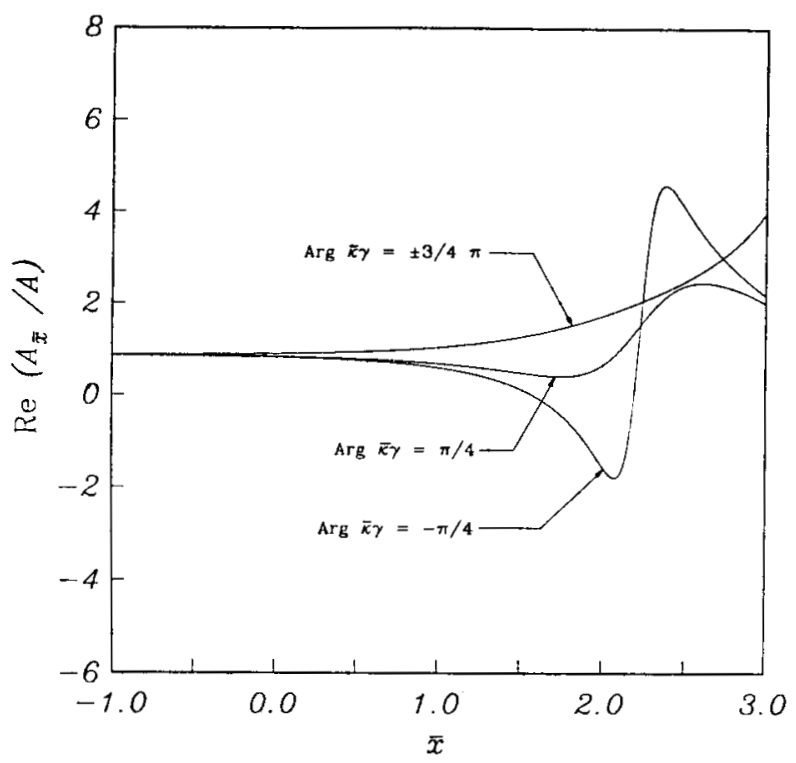

FIGURE 4. - GROWTH RATE Re A $\bar{x} / A$ VERSUS SCALED STREAMWISE COORDIMATE $\bar{x}$ FOR $\theta=\pi / 4$ AND ARG. $\bar{k}=-\pi / 6$.

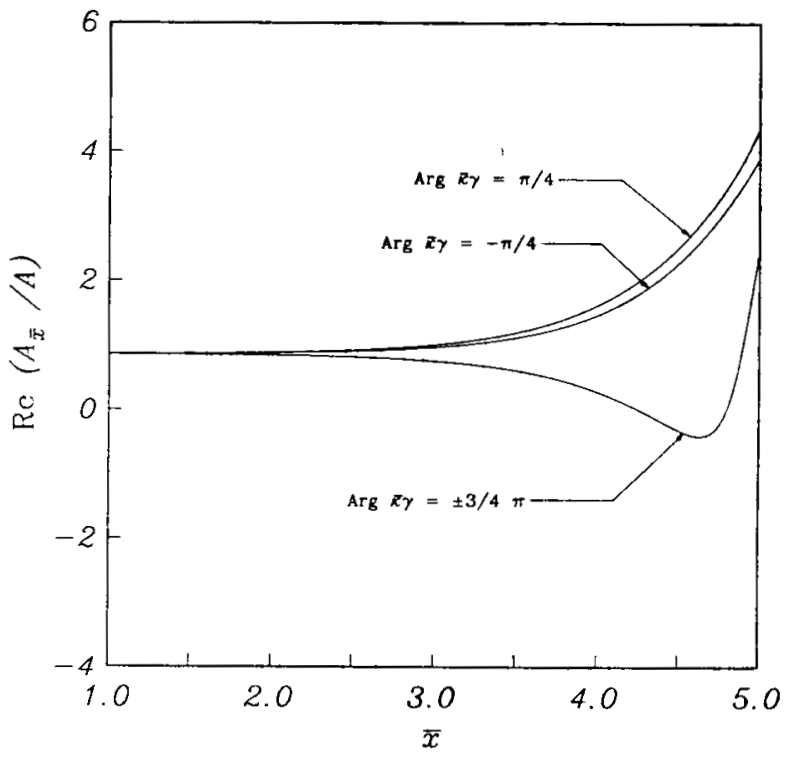

FIGURE 3. - GROWTH RATE Re $A_{\bar{x}} / A$ VERSUS SCALED STREAMWISE COORDINATE $\bar{x}$ FOR $\theta=\pi / 8$ AND ARG. $\bar{k}=-\pi / 6$.

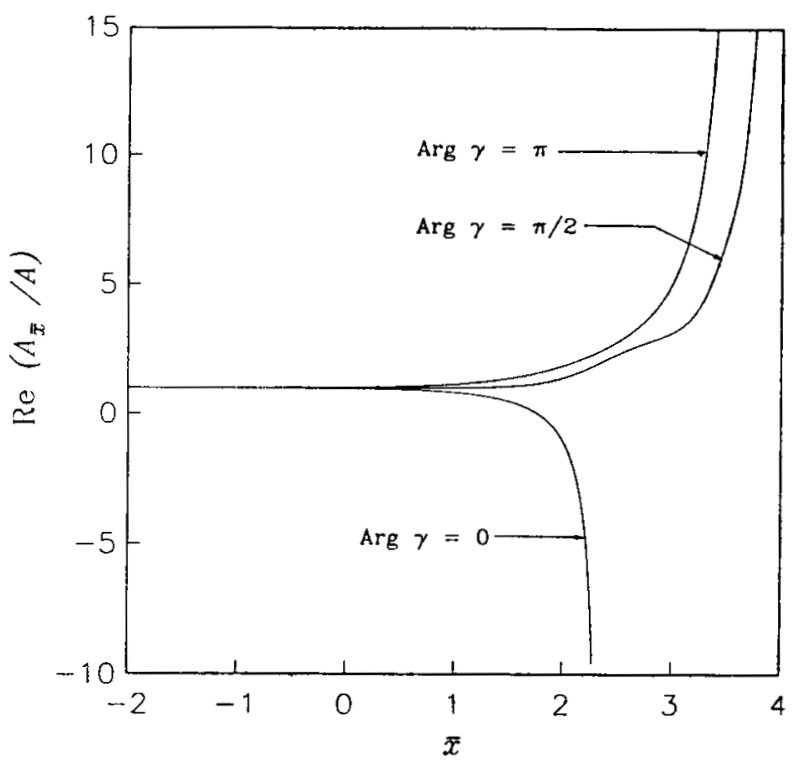

FIGURE 5. - GROWTH RATE Re A-/A VERSUS SCALED STREAMWISE COORDINATE $\bar{x}$ FOR $\theta=\bar{\pi} / 4$ AND ARG. $\bar{k}=0$. 


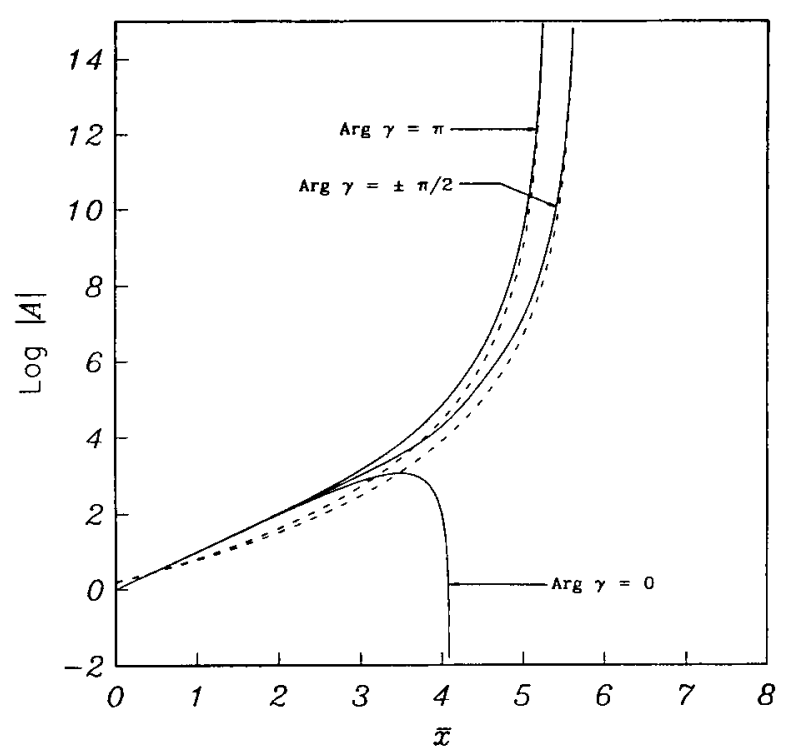

(a) $\theta=\pi / 8$.

FIGURE 6. - AMPLITUDE LOG $|A|$ VERSUS SCALED STREAMHISE COORDIMATE $\bar{X}$ FOR ARG, $\bar{K}=0$.

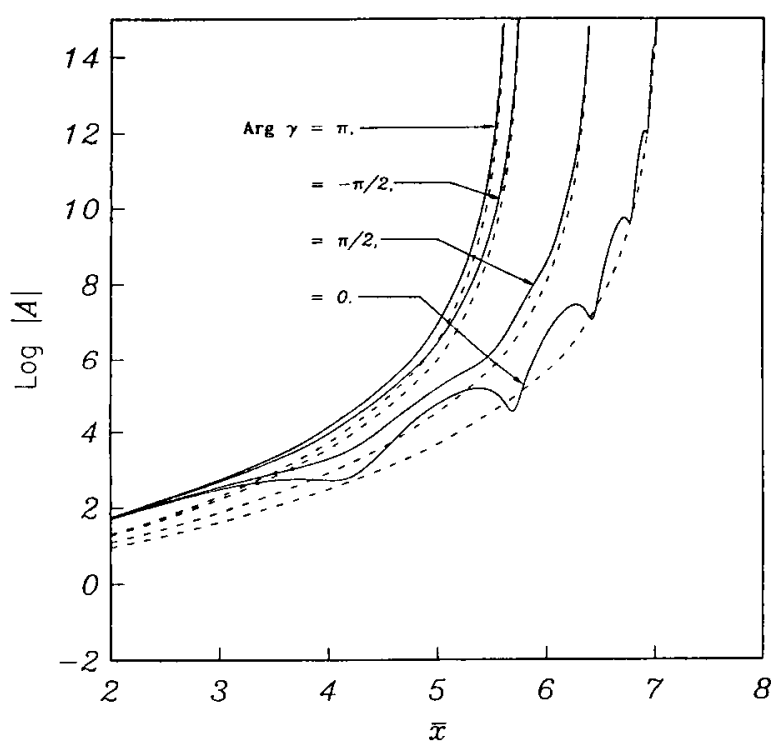

(a) $\theta=\pi / 8$.

FIGURE 7. - AMPLITUDE LOG $|A|$ VERSUS SCALED STREAMWISE COORDINATE $\bar{x}$ FOR ARG. $\bar{k}=-\pi / 6$.

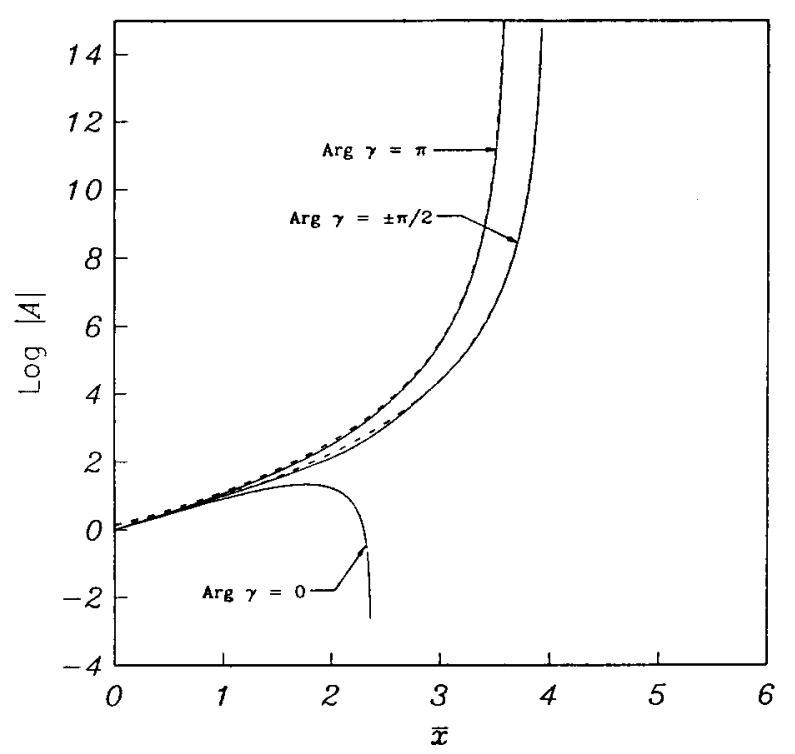

(b) $\theta=\pi / 4$.

FIGURE 6. - CONCLUDED.

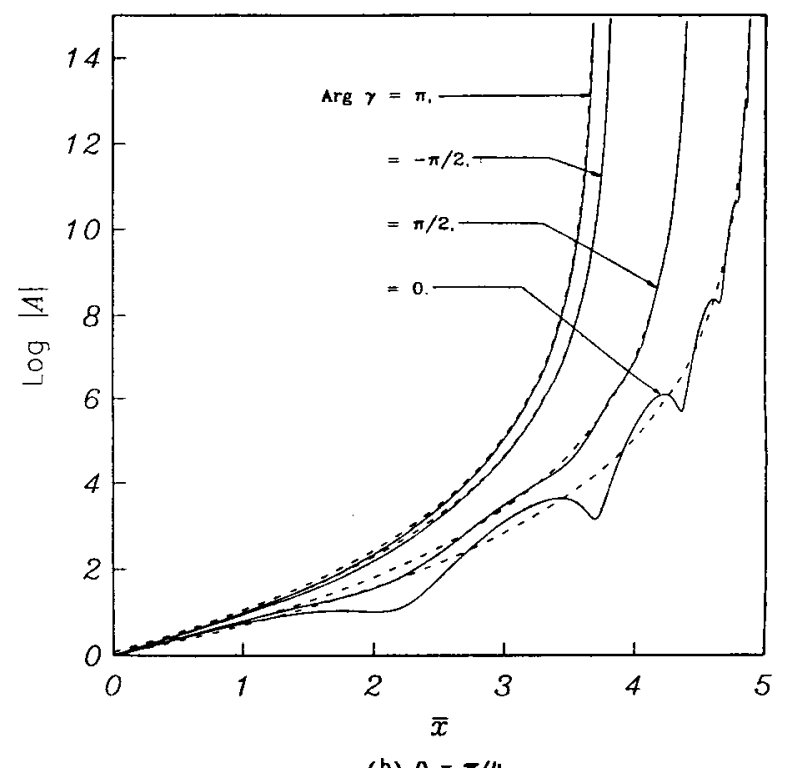

(b) $\theta=\pi / 4$.

FIGURE 7. - CONCLUDED. 


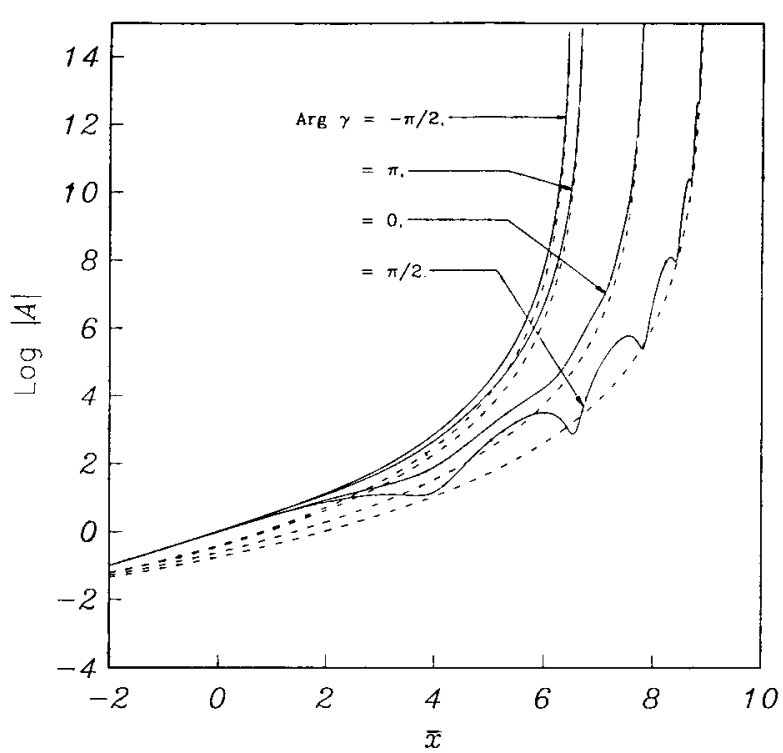

(a) $\theta=\pi / 8$.

FIGURE 8. - AMPLITUDE LOG $\mid$ A| VERSUS SCALED STREAMWISE COORDINATE $\bar{x}$ FOR ARG. $\bar{K}=-\pi / 3$.

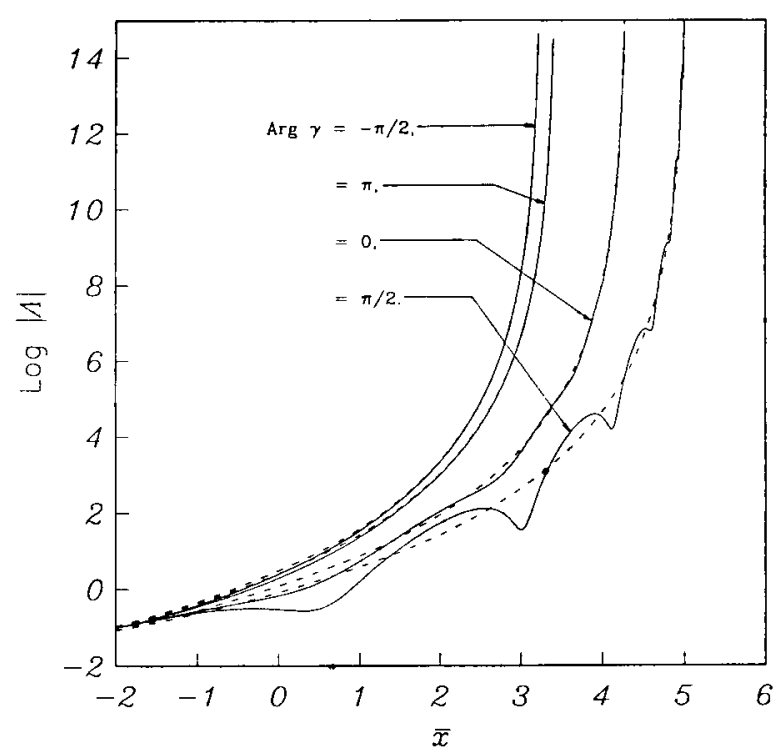

(b) $\theta=\pi / 4$.

FIGURE 8. - CONCLUDED.

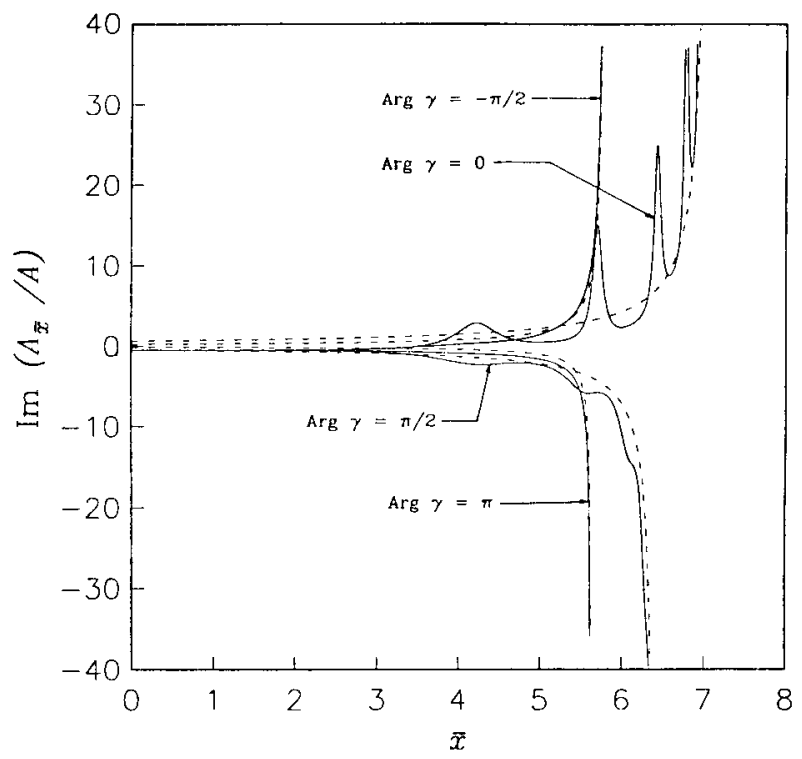

FIGURE 9. - PHASE Im A-/A VERSUS SCALED STREAMWISE COORDINATE $\overline{\mathrm{x}}$ FOR $\theta=\pi / 8$ AND ARG, $\bar{k}=-\pi / 6$. 


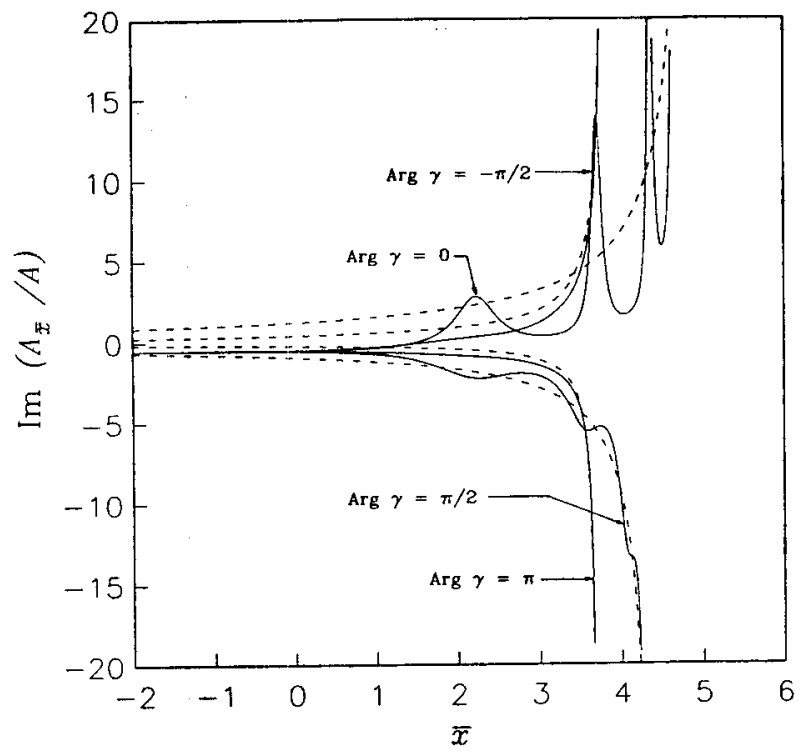

FIGURE 10. - PHASE Im $A_{\bar{x}} / A$ VERSUS SCALED STREAMWISE COORDINATE. $\bar{x}$ FOR $\theta=\pi / 4$ AND ARG. $\vec{k}=-\pi / 6$.

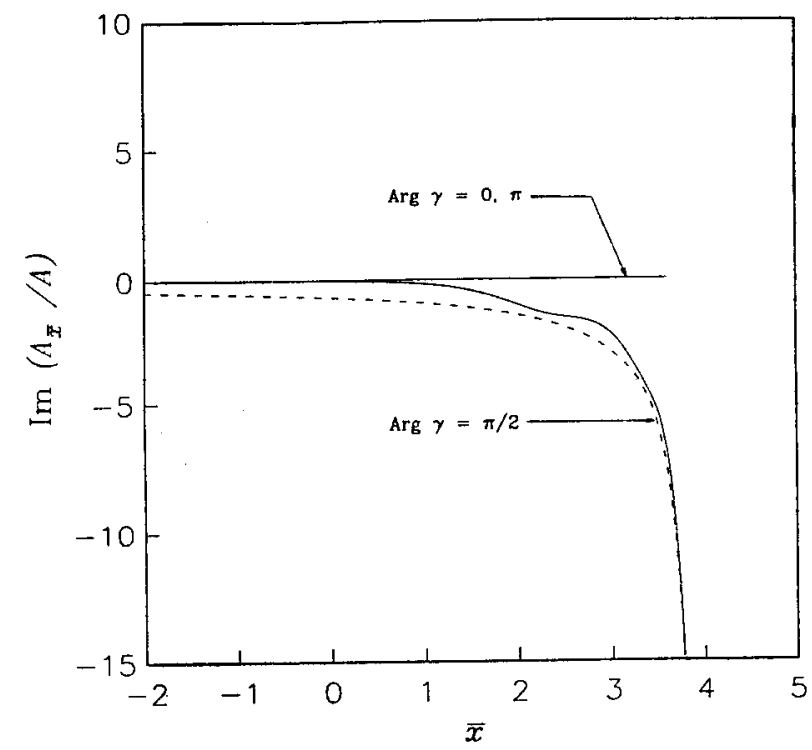

FIGURE 11. - PHASE Im $A_{\bar{x}} / A$ VERSUS SCALEd STREAMWISE COORDINATE $\bar{x}$ FOR $\theta=\pi / 4$ AND ARG. $\bar{k}=0$. 


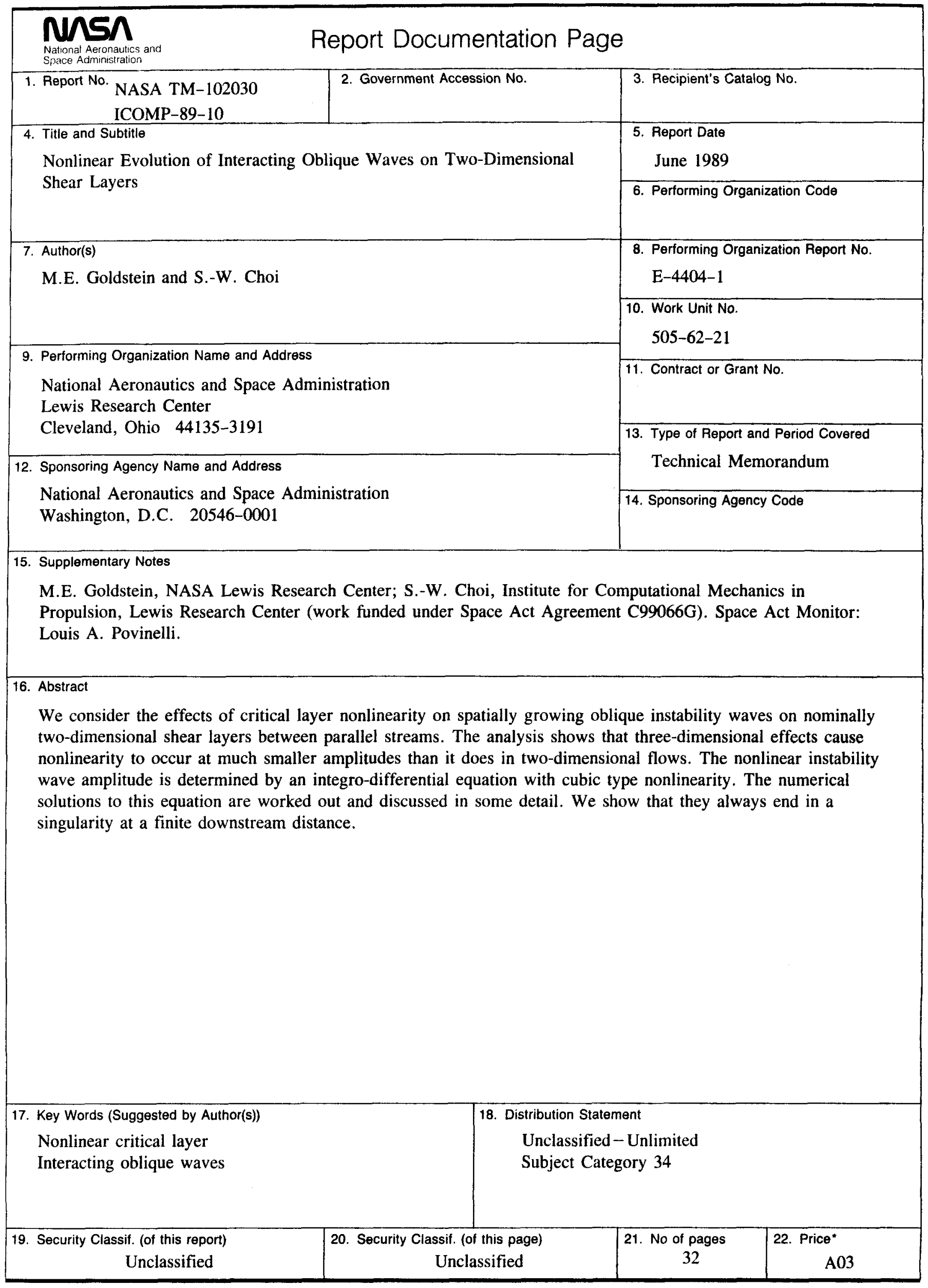

